FERMILAB-FN-0760-AD

\title{
TRANSVERSE INSTABILITY AT THE RECYCLER RING
}

\author{
K.Y. Ng \\ Fermi National Accelerator Laboratory, P.O. Box 500, Batavia, IL 60510
}

(July 14, 2004)

\begin{abstract}
Sporadic transverse instabilities have been observed at the Fermilab Recycler Ring leading to increase in transverse emittances and beam loss. The driving source of these instabilities has been attributed to the resistive-wall impedance with space-charge playing an important role in suppressing Landau damping. Growth rates of the instabilities are computed. Remaining problems are discussed.
\end{abstract}




\section{INTRODUCTION}

The Fermilab Recycler Ring is a ring to store, accumulate and stochastically cool antiprotons. Since the beginning of 2004, transverse instabilities have been reported in the antiproton beam with the signature of a sudden increase in the transverse emittances and a small loss in beam intensity.

The first such documented transverse instability was observed on February 19, 2004. [2] An antiproton beam of intensity $126 \times 10^{10}$ and length $7.3 \mu$ s was stored in the Recycler Ring between two barrier waves. The horizontal and vertical 95\% normalized emittances were cooled stochastically to about $7 \pi \mathrm{mm}-\mathrm{mr}$. The horizontal and vertical emittances were roughly the same because the residual horizontal and vertical betatron tunes were close resulting in strong horizontal and vertical coupling. As seen in the left plot of Fig. 1, the emittances took a sudden jump to about $15 \pi \mathrm{mm}-\mathrm{mr}$ at about 12:00 noon. At the same time, as shown in the right plot of Fig. 1, there was a small beam loss of about $1 \times 10^{10}$. When the record of the Main Injector, that shares the same tunnel with the Recycler Ring, was traced, it was found that the Main Injector changed its ramp pattern a few hours earlier.
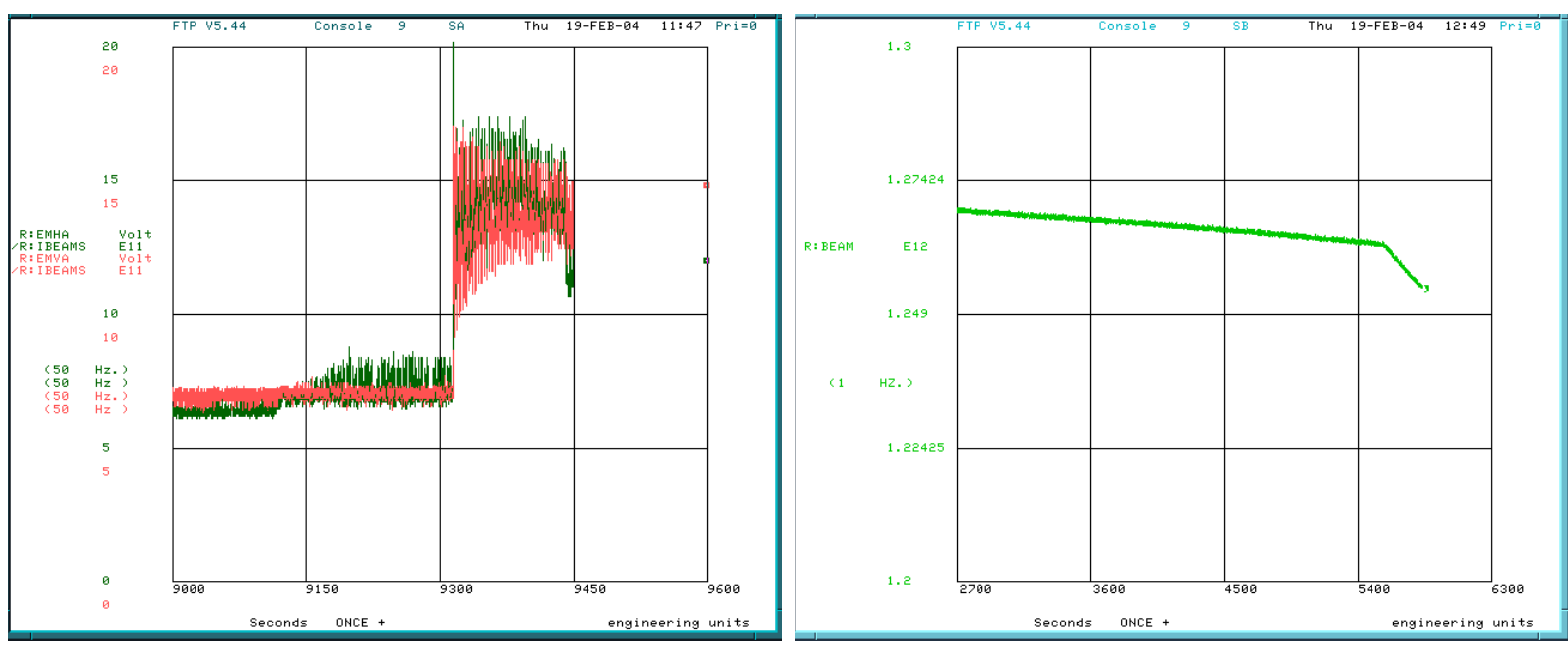

Figure 1: (color) An antiproton beam of intensity $126 \times 10^{10}$ and length $7.3 \mu$ s was seen on February 19 to suffer a transverse instability with a sudden emittance growth (left) and a slight beam loss (right).

Some common features of these instabilities are:

1. The beams are all antiprotons of various lengths, from $5.28 \mu \mathrm{s}$ to $9.3 \mu \mathrm{s}$, bunched in a 
barrier bucket.

2. The instabilities occurred with beam intensities either about $30 \times 10^{10}$ or $125 \times 10^{10}$

3. The transverse $95 \%$ normalized emittances of the beam have been cooled to less than $4 \pi$ mm-mr (except the one on February 19).

4. Every instability appears to be preceded at least one hour or more by a reduction in the ramp cycle of the Main Injector. The stray fields of the Main Injector during ramping affect the orbit of the Recycler Ring. Although there is a correction mechanism using beam-position monitors and bump magnets, the compensation has never been complete, especially when the ramping pattern of the Main Injector is changed. The closed orbit of the Recycler beam will be pushed inwards and outwards according to the pulsation of the partially compensated stray fields.

It has been suggested that these instabilities are related to ions trapped inside the antiproton beam, although no direct verification has been made. Various analysis has been made in this direction without any decisive conclusion. [1]

Since the instabilities do not occur very often, to study them, similar instabilities were induced, [3, 4] although it is unclear whether what were induced are the same instabilities that were observed before. The methods of inducing transverse instabilities include:

1. Turning off rf. When the rf is off, the beam will spill into the beam gap eliminating the possibility of ion clearing.

2. Turning on/off cooling. On the one hand, intense stochastic cooling increases the transverse density of the beam and may lead to instability. On the other hand, turning off stochastic cooling may cease to help stabilize a transverse instability if the instability growth rate is extremely slow.

3. The quadrupole-correction loop (QCL). The two quadrupole buses of the Main Injector leave a net current of $\sim 100 \mathrm{~A}$ around the circumference of the ring. The dipole field created by this difference current is usually compensated by passing an equal and opposite current through a quadrupole-correction loop (QCL) around the ring. Therefore pulsing the QCL when the Main Injector is not ramping or turning off the QCL while the Main Injector is ramping will mimic the sudden change in ramping pattern of the Main Injector. 
4. Decreasing chromaticity to zero. The Recycler antiproton beam has not been stable at low chromaticities if it is very intense. Thus reducing the nominal chromaticities $\xi_{x}$ and $\xi_{y}$ to zero may help induce an instability.

On June 9, an experiment was performed on a $28 \times 10^{10}$ antiproton beam of length $3.5 \mu \mathrm{s}$ by turning off the QCL and reducing the vertical chromaticity to zero. [3] An instability was induced with a blowup in $95 \%$ normalized vertical emittance from $\epsilon_{N 95}=3.0 \pi \mathrm{mm}-\mathrm{mr}$ to about $10 \pi \mathrm{mm}-\mathrm{mr}$ and a small beam loss of $3 \times 10^{10}$. During the instability, the difference signals were collected at a split-tube vertical beam-position monitor at location VP522, where the horizontal and vertical betatron functions are, respectively, $47.6 \mathrm{~m}$ and $16.7 \mathrm{~m}$. The Fourier transform up to roughly the first 70 revolution harmonics is shown in Fig. 2. The slow roll-off of the lower betatron sidebands up to the 70th revolution harmonic $(\sim 7.2 \mathrm{MHz})$ indicates that the instability might be driven by resistive-wall impedance. On July 9 of 2004, a similar experiment was performed on a proton beam by reducing the chromaticity. [4] The proton beam was prepared as coasting by turning off the rf barrier voltages. A very similar transverse instability was induced when the beam intensity was larger than $40 \times 10^{10}$ and the transverse emittances were reduced by scraping, because the proton beam, traveling in the opposite direction of the antiprotons, could not take advantage of the stochastic cooling. This proton experiment indicates very clearly that these instabilities were probably driven by the resistive-wall impedance rather than by trapped ions.

In this paper, we would like to compute the growth rates of the instabilities, compare them with experimental observation, and discuss some remaining problems. We end this section by quoting in Table I some relevant properties of the Fermilab Recycler ring, which is a ring with permanent gradient magnets and quadrupoles.

Table I: Some relevant properties of the Fermilab Recycler Ring.

\begin{tabular}{lr}
\hline Circumference $(\mathrm{m})$ & $\sim 3319.419$ \\
Kinetic energy $(\mathrm{GeV})$ & $\sim 8.00$ \\
Revolution frequency $(\mathrm{kHz})$ & 89.812 \\
Revolution period $(\mu \mathrm{s})$ & 11.134 \\
Slip factor $(\eta)$ & -0.008511 \\
Horizontal betatron tune & 25.425 \\
Vertical betatron tune & 24.415 \\
\hline
\end{tabular}



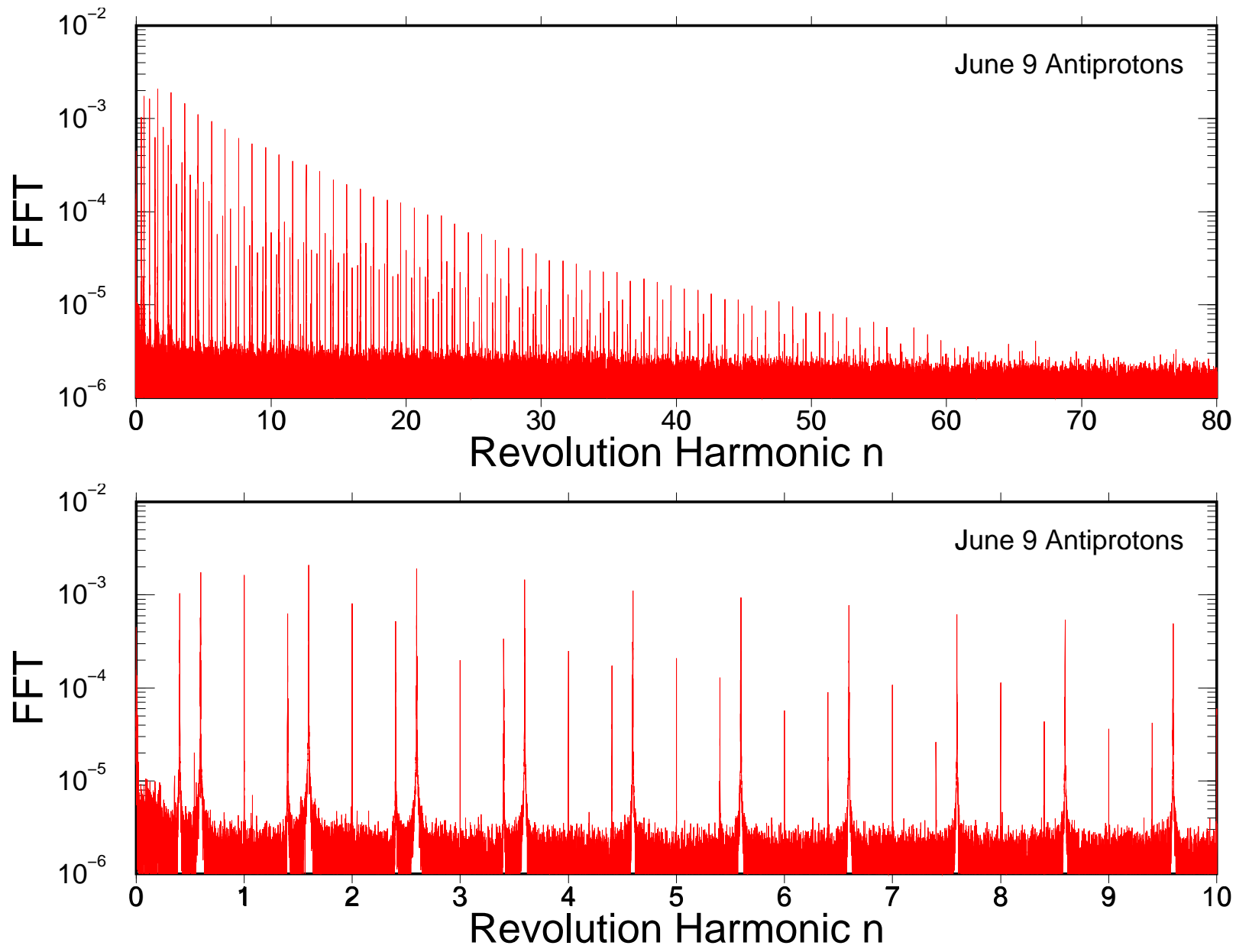

Figure 2: (color) Top: Betatron sidebands of the first 80 rotation harmonics monitored at the splittube beam-position monitor VP522 during the instability induced on June 9, 2004. The antiproton beam that went into instability had an initial intensity of $28 \times 10^{10}$ and the initial emittance were $\epsilon_{N 95}=3.0 \pi \mathrm{mm}-\mathrm{mr}$. Bottom: Zoom-in view of the first 10 harmonics. The pre-amp on VP522 monitor had flat response from $10 \mathrm{kHz}$ to $10 \mathrm{MHz}$. 


\section{DISPERSION RELATION}

The equation governing the vertical displacement $y$ of a beam particle of mass $m$ and energy $E=\gamma m c^{2}$ is

$$
\frac{d^{2} y}{d t^{2}}+\omega_{y 0}^{2} y=\frac{1}{\gamma m}\left[F_{\mathrm{coh}}^{\prime}\langle y\rangle+F_{\mathrm{inc}}^{\prime}(y-\langle y\rangle)\right]
$$

where the first term on the right-side is responsible for coherent betatron tune shift and the second term incoherent betatron tune shift. In above, $\nu_{y 0}=\omega_{y 0} / \omega_{0}$, with $\omega_{0}$ being the angular revolution frequency, is the bare vertical betatron tune, which is adjusted to the incoherent betatron tune $\nu_{y}=\omega_{y} / \omega_{0}$ when combined with the term $F_{\text {inc }}^{\prime} y$ on the right side. What is left on the right side

$$
F_{\text {wake }}^{\prime}\langle y\rangle=\left(F_{\text {coh }}^{\prime}-F_{\text {inc }}^{\prime}\right)\langle y\rangle
$$

is the dipole wake force acting on the particle when the center of the beam is displaced by $\langle y\rangle$. The vertical particle displacement $y$ is a function of $(s, t)$ where $s$ is the distance along the ring. For a coasting beam, we can denote the snapshot dipole current of the center of the beam by the ansatz,

$$
I\langle y(s, t)\rangle=I\langle\tilde{y}\rangle e^{i n s / R-i \Omega t},
$$

where $\langle\tilde{y}\rangle$ denotes the amplitude and is time-independent. The growth is attributed to the imaginary part of the collective angular frequency $\Omega$, to be determined by the solution of the equation of motion. In above, $n$ is a revolution harmonic and $R$ is the mean radius of the ring. The ansatz just postulates that the revolution harmonics are not related and can be studied individually. At a fixed location $s$ of the ring, a test particle experiences the wake force left by the dipole current averaged over one revolution turn,

$$
F_{\text {wake }}^{\prime}\langle y(s, t)\rangle=-\frac{e}{2 \pi R} \int_{-\infty}^{t} v d t^{\prime} W_{1}\left(v t-v t^{\prime}\right) I\left\langle y\left(s, t^{\prime}\right)\right\rangle=\frac{i e \beta^{2} c I\langle y(s, t)\rangle Z_{1}^{V}(\Omega)}{2 \pi R}
$$

where $v=\beta c$ is the nominal velocity of the particles in the beam, $W_{1}$ is the dipole wake function, and $Z_{1}^{V}$ the vertical dipole impedance.

Now let us come back to the beam particle described by Eq. (2.1). If it passes the location $S$ at time $t=0$, its location at a later time $t$ is $s=S+v t$, and the equation of motion becomes

$$
\frac{d^{2} y}{d t^{2}}+\omega_{y}^{2} y=\frac{i e I c Z_{1}^{V}}{E T_{0}}\langle\tilde{y}\rangle e^{i n S / R-i\left(\Omega-n \omega_{0}\right) t}
$$


where $T_{0}=2 \pi / \omega_{0}$ is the nominal revolution period of the beam and $E$ is the nominal energy. The right-side can be re-written conveniently as $-2 \omega_{y} \Delta \omega_{y}\langle y(s, t)\rangle$, where

$$
\Delta \nu_{y}=\frac{\Delta \omega_{y}}{\omega_{0}}=-\frac{i e I c Z_{1}^{V}}{4 \pi \omega_{y} E}
$$

represents a tune shift. Sometimes it is loosely called the coherent tune shift.* But actually, according to Eq. (2.2), it is the difference of the coherent and incoherent tune shifts.

This force-driven differential equation can be solved easily, giving the solution

$$
\left[\omega_{y}^{2}-\left(\Omega-n \omega_{0}\right)^{2}\right] y(s, t)=-2 \omega_{y} \Delta \omega_{y}\langle\tilde{y}\rangle e^{i n S / R-i\left(\Omega-n \omega_{0}\right) t},
$$

For small perturbation, there are two solutions for the coherent frequency $\Omega \approx n \omega_{0} \pm \omega_{y}$. For a coasting beam, when we allow for both positive and negative revolution harmonics, the same physical conclusion will be achieved when we choose either $\Omega \approx n \omega_{0}+\omega_{y}$ or $n \omega_{0}-\omega_{y}$. This is because (1) the beam spectra of the two choices are related by symmetry and (2) $Z_{1}^{V}(\omega)$ has definite symmetry about $\omega=0$. We choose the convention of upper sidebands, which leads us to the approximation,

$$
\omega_{y}^{2}-\left(\Omega-n \omega_{0}\right)^{2}=\left(\omega_{y}-\Omega+n \omega_{0}\right)\left(\omega_{y}+\Omega-n \omega_{0}\right) \approx 2 \omega_{y}\left(\omega_{y}-\Omega+n \omega_{0}\right)
$$

The solution of Eq. (2.7) becomes

$$
y(s, t)=-\frac{\Delta \omega_{y}\langle\tilde{y}\rangle}{\omega_{y}-\Omega+n \omega_{0}} e^{i n S / R-i\left(\Omega-n \omega_{0}\right) t}
$$

Self-consistency with the ansatz of Eq. (2.3) leads to the dispersion relation,

$$
1=\Delta \omega_{y} \int_{-\infty}^{\infty} \frac{\rho\left(\omega_{y}\right) d \omega_{y}}{\Omega-n \omega_{0}-\omega_{y}}
$$

where we have introduced the distribution density $\rho\left(\omega_{y}\right)$ of the particles in the beam, which is normalized to unity when integrated over $\omega_{y}$. Solution of Eq. (2.10) gives the collective betatron frequency $\Omega$ of the beam as observed at a fixed location at the ring. It can be verified easily that when there is no spread in betatron frequency among the beam particles, $\Delta \omega_{y}=\Omega-n \omega_{0}-\omega_{y}$ indeed represents a dynamic contribution of the coherent betatron tune shift of the beam.

*The coherent tune shift is defined as the shift of the coherent betatron tune from the bare betatron tune. The shift here, however, is from the incoherent betatron tune to the coherent betatron tune, which is the dynamical part of the coherent tune shift. 
To solve the dispersion relation, let us consider the situation of Landau damping coming from energy spread only. For a particle with momentum offset $\delta$,

$$
\omega_{y}+n \omega_{0}=\bar{\omega}_{y}+n \bar{\omega}_{0}+\left[\xi_{y}-\eta\left(n+\bar{\nu}_{y}\right)\right] \omega_{0} \delta
$$

where $\xi_{y}$ and $\eta$ denote, respectively, the vertical chromaticity and slip-factor of the ring. In above, $\bar{\omega}_{y}, \bar{\omega}_{0}$, and $\bar{\nu}_{y}$ denote, respectively, the nominal angular betatron frequency, nominal angular revolution frequency, and nominal vertical betatron tune of the beam particles. However, for the sake of convenience, these overhead bars will be omitted in below. As a short-hand notation, we introduce the effective chromaticity

$$
S_{y}=\xi_{y}-\eta\left(n+\nu_{y}\right)
$$

The dispersion relation now takes the form ${ }^{\dagger}$

$$
1=\frac{\Delta \omega_{y}}{\left|S_{y}\right| \omega_{0}} \int_{-\infty}^{\infty} \frac{\tilde{\rho}(\delta) d \delta}{\hat{\Omega}-\delta},
$$

with

$$
\hat{\Omega}=\frac{\Omega-n \omega-\omega_{y}}{\left|S_{y}\right| \omega_{0}},
$$

where $\tilde{\rho}(\delta)$ is the distribution density of the beam particles in momentum spread, and is normalized to unity when integrated over $\delta$. Although the effective chromaticity $S_{y}$ can be positive or negative, we choose to normalize the eigen-frequency by $\left|S_{y}\right|$, so that $\operatorname{Im} \hat{\Omega}>0$ always implies an instability.

The integral in the dispersion relation must be an analytic function of $\hat{\Omega}$ because the impedance $Z_{1}^{V}$ is an analytic function of the eigen-frequency $\Omega$. With the path of integration along the real axis, however, it is obvious that the integral is discontinuous across $\mathcal{I} m \hat{\Omega}=0$. This is because

$$
\int_{-\infty}^{\infty} \frac{\tilde{\rho}(\delta)}{x \pm i \epsilon-z} d z=\wp \int_{-\infty}^{\infty} \frac{\tilde{\rho}(\delta)}{x-z} d z \mp i \pi \frac{\rho(x)}{x}
$$

where $x$ is real, $\epsilon$ is positive but infinitesimal, and $\wp$ denotes the principal value. Analyticity can be achieved by continuing the upper $\hat{\Omega}$ half plane into the lower $\hat{\Omega}$ half plane. This analytic continuation is accomplished by distorting the path of integration from $\delta=(-\infty, \infty)$ to the path $C$ so that it always goes below the pole $\delta=\hat{\Omega}$, as illustrated in Fig. 3. In order

$$
\begin{aligned}
& { }^{\dagger} \text { We must be more careful when } S_{y}<0 \text {. We have } \\
& 1=\frac{\Delta \omega_{y}}{\left|S_{y}\right| \omega_{0}} \int_{-\delta=-\infty}^{-\delta=\infty} \frac{\rho\left(\bar{\omega}_{y}-\left|S_{y}\right| \omega_{0} \delta\right) d\left(-\left|S_{y}\right| \omega_{0} \delta\right)}{\hat{\Omega}+\delta}=\int_{\delta=-\infty}^{\delta=\infty} \frac{\rho\left(\bar{\omega}_{y}-\left|S_{y}\right| \omega_{0} \delta\right)\left|S_{y}\right| \omega_{0} d \delta}{\hat{\Omega}-\delta} \int_{\delta=-\infty}^{\infty} \frac{\tilde{\rho}(\delta) d \delta}{\hat{\Omega}-\delta},
\end{aligned}
$$

where the distribution in $\delta$ is $\tilde{\rho}(\delta)=\rho\left(\bar{\omega}_{y}-\left|S_{y}\right| \omega_{0} \delta\right)\left|S_{y}\right| \omega_{0}$. 


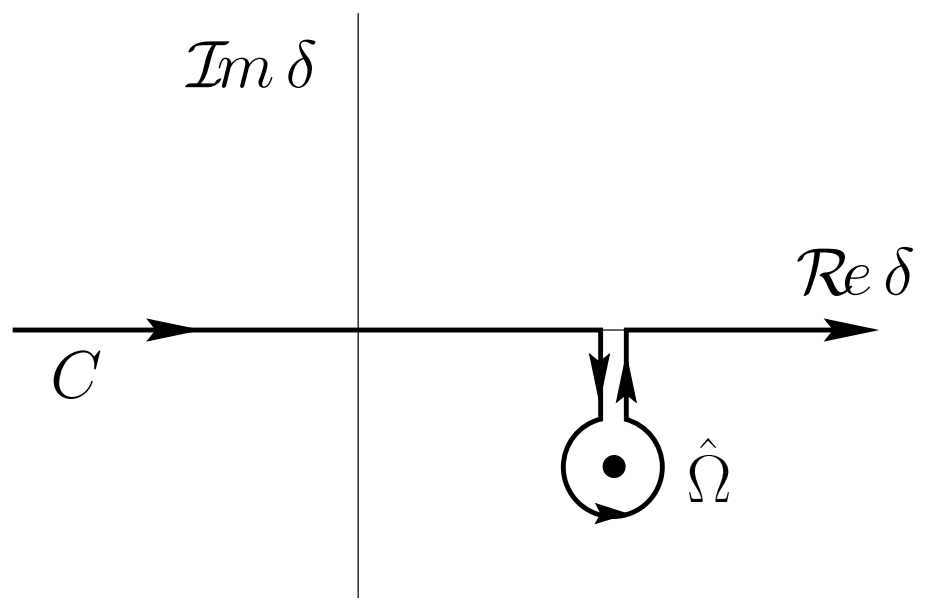

Figure 3: To satisfy causality and analyticity, the integration contour $C$ must be chosen to go below the pole $\delta=\hat{\Omega}$.

to incorporate the initial condition that the driving force has not been in existence since $t=-\infty$, the eigen-frequency always has a infinitesimal positive imaginary part; or instead of $\Omega$, we should write $\Omega+i \epsilon$. This explains why we continue the upper $\hat{\Omega}$ half plane into the lower $\hat{\Omega}$ half plane instead of continuing the lower half plane into the upper half plane.

For the Gaussian distribution density with the rms momentum offset $\sigma_{\delta}$,

$$
\tilde{\rho}(\delta)=\frac{1}{\sqrt{2 \pi} \sigma_{\delta}} e^{-\delta^{2} /\left(2 \sigma_{\delta}^{2}\right)}
$$

the dispersion relation becomes

$$
1=\frac{\Delta \omega_{y}}{\left|S_{y}\right| \omega_{0}} \frac{1}{\sqrt{2 \pi} \sigma_{\delta}} \int_{C} \frac{e^{-z^{2}} d z}{z_{1}-z}=-\frac{i \pi \Delta \omega_{y}}{\sqrt{2 \pi} \sigma_{\delta}\left|S_{y}\right| \omega_{0}} w\left(z_{1}\right),
$$

where $z_{1}=\hat{\Omega} /\left(\sqrt{2} \sigma_{\delta}\right)$ and $w\left(z_{1}\right)$ is the complex error function.

Let us introduce the traditional reduced vertical dipole impedance $(U, V)$ by

$$
U+i V=-\frac{i \pi \Delta \omega_{y}}{\sqrt{2 \pi} \sigma_{\delta}\left|S_{y}\right| \omega_{0}}=-\frac{e I_{0} \beta^{2} c Z_{1}^{\perp}}{4 \sqrt{2 \pi} \nu_{y} \omega_{0} \sigma_{E}\left|S_{y}\right|} .
$$

where the substitution of $\Delta \omega_{y}$ from Eq. (2.6) has been made. Thus given the vertical transverse impedance, we can solve the dispersion relation

$$
U+i V=\frac{1}{w\left(z_{1}\right)}
$$


to obtain the growth rate

$$
\frac{1}{\tau}=\operatorname{Im} \Omega=\sqrt{2} \sigma_{\delta}\left|S_{y}\right| \omega_{0} \operatorname{Im} z_{1}
$$

\section{IMPEDANCES OF THE RECYCLER RING}

\subsection{Resistive-Wall Impedance}

For a cylindrical beam pipe of radius $b$, the monopole longitudinal impedance and dipole transverse impedance are

$$
\left.Z_{0}^{\|}\right|_{\mathrm{cyl}}=[1-i \operatorname{sgn}(\omega)] \frac{R \rho_{s}}{b \delta_{s}},\left.\quad Z_{1}^{\perp}\right|_{\mathrm{cyl}}=\left.\frac{2 c}{b^{2}} \frac{Z_{0}^{\|}}{\omega}\right|_{\mathrm{cyl}},
$$

where $\delta_{s}$ is the skip-depth into the walls of the beam pipe and $\rho_{s}$ is the resistivity.

For the elliptical beam pipe, the monopole longitudinal impedance and the dipole horizontal and vertical impedances can be written as

$$
Z_{0}^{\|}=\left.Z_{0}^{\|}\right|_{\mathrm{cyl}} F_{0}, \quad Z_{1}^{H}(\omega)=\left.Z_{1}^{\perp}\right|_{\mathrm{cyl}} F_{H}, \quad Z_{1}^{V}(\omega)=\left.Z_{1}^{\perp}\right|_{\mathrm{cyl}} F_{V}
$$

where the elliptical form factors can be expressed in terms of elliptical functions according to Ref. [5]. The beam pipe of the Recycler Ring is made of stainless steel of resistivity $\rho_{s s}=7.4 \times 10^{-7} \Omega \mathrm{m}$, and elliptical in cross section with horizontal and vertical diameters, respectively, $2 a=3.75^{\prime \prime}$ and $2 b=1.75^{\prime \prime}$. The form factors are $F_{0}=0.9591, F_{H}=0.4501$, and $F_{V}=0.8369$. Thus we obtain the resistive wall impedances:

$$
\begin{aligned}
& Z_{0}^{\|}=(1-i) 11.68 n^{1 / 2} \Omega \\
& Z_{1}^{H}=(1-i) 11.79\left|n-\left[\nu_{x}\right]\right|^{-1 / 2} \mathrm{M} \Omega / \mathrm{m} \\
& Z_{1}^{V}=(1-i) 21.92\left|n-\left[\nu_{y}\right]\right|^{-1 / 2} \mathrm{M} \Omega / \mathrm{m}
\end{aligned}
$$

where, for the transverse impedances, $n=1,2,3, \cdots$ denotes the excitation of the lower betatron sidebands $n-\left[\nu_{y}\right]$, which are also commonly known as the $(n-Q)$ lines.

We see in Fig. 2 that the instability on June 9 was excited up to the revolution harmonic

$n \approx 70$ only. If the instability was excited mainly by the resistive wall impedance, this implies 
that the beam is unstable only when $Z_{1}^{V} \gtrsim 21.9 / \sqrt{70-\left[\nu_{y}\right]}=2.63 \mathrm{M} \Omega / \mathrm{m}$. From Eq. (2.18) the actual driving force is proportional to

$$
F=\frac{\operatorname{Re} Z_{1}^{V}}{\left|S_{y}\right|} .
$$

If the instability on June 9 occurred at $\xi_{y}=0$ and is driven by the resistive-wall impedance, then the minimum driving force for an observed excitation will become

$$
F_{\min }=\frac{2.63}{|\eta|\left(n-\left[\nu_{y}\right]\right)} \mathrm{M} \Omega / \mathrm{m}=4.29 \mathrm{M} \Omega / \mathrm{m} .
$$

\subsection{Space-Charge Impedance}

The transverse space-charge impedance is given by

$$
\left.Z_{1}^{V}\right|_{\mathrm{spch}}=i \frac{Z_{0} R}{\gamma^{2} \beta^{2} a^{2}}
$$

where $Z_{0} \approx 376.7 \Omega$ is the free-space impedance, $a$ is the radius of the beam if the transverse distribution is uniform. The average vertical beta-function is $\beta_{y}=R / \nu_{y}$. In terms of the $95 \%$ normalized emittance $\epsilon_{N 95}$, the rms beam radius for a bi-Gaussian transverse distribution is

$$
\sigma_{y}=\sqrt{\frac{\epsilon_{N 95} \beta_{y}}{6 \beta \gamma}} .
$$

It can be shown easily [6] that the equivalent-uniform-distribution beam radius of a biGaussian distribution is $a=\sqrt{2} \sigma_{y}$. Thus at $\epsilon_{N 95}=3 \pi \mathrm{mm}-\mathrm{mr}$,

$$
Z_{1}^{V}=i \frac{3 Z_{0} \nu_{y}}{\gamma \beta \epsilon_{N 95}}=971 \Omega / \mathrm{m}
$$

which is very much larger than the resistive-wall impedance in magnitude. There is also an image contribution which reads

$$
\left.Z_{1}^{\perp}\right|_{\text {image }}=-i \frac{Z_{0} R}{\gamma^{2} \beta^{2} b^{2}}
$$

for a round beam pipe of radius $b$. Using $b=0.875^{\prime \prime}$, the contribution is only $-i 0.11 \mathrm{M} \Omega / \mathrm{m}$, and can therefore be safely neglected. 


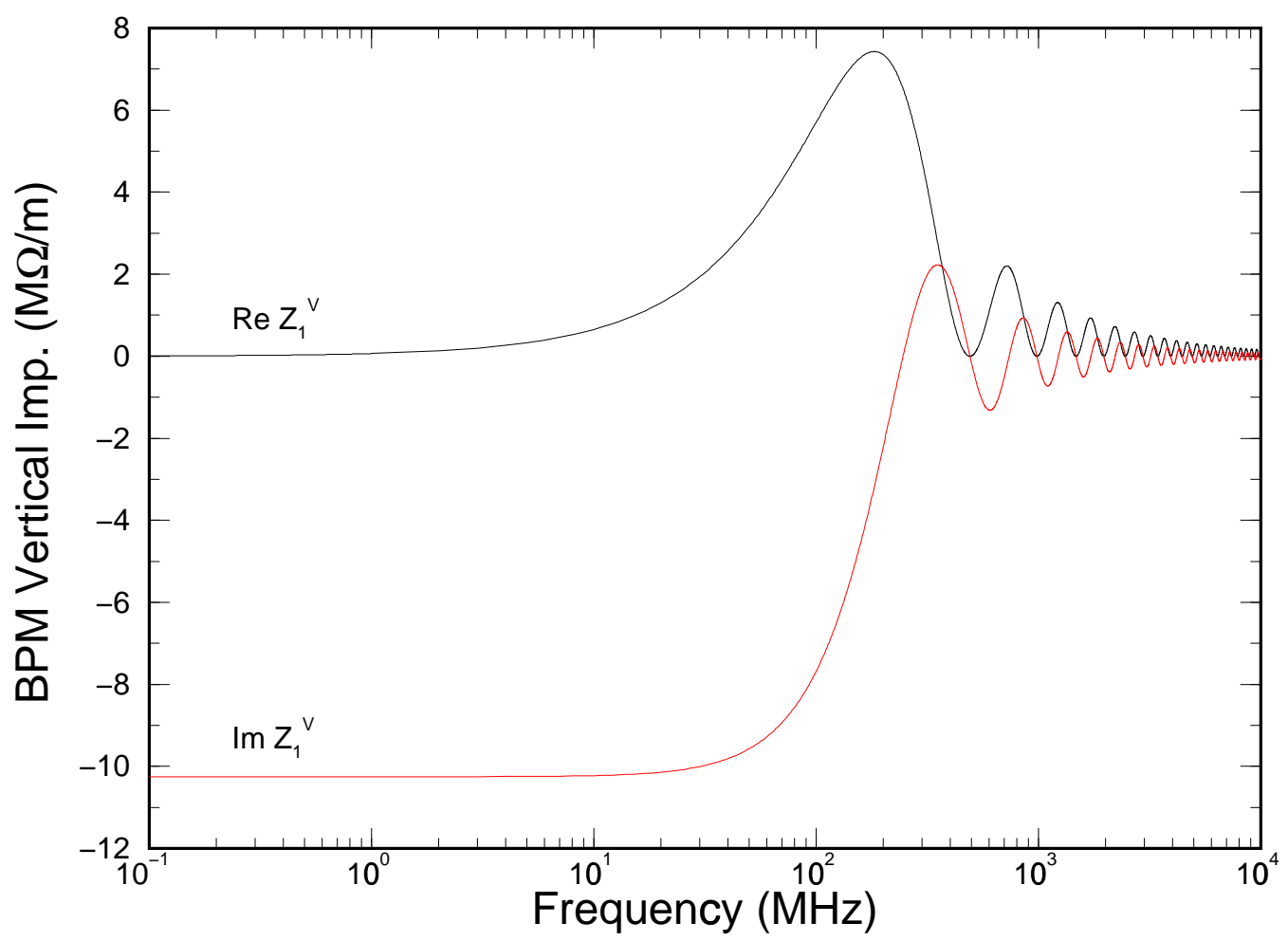

Figure 4: (color) Vertical dipole impedance of the 205 sets of vertical BPM in the Recycler Ring.

\subsection{Beam-Position Monitors}

If we approximate the split-can BPMs as cylindrical strip lines of length $\ell=12^{\prime \prime}$ with covering angle $\phi_{0} \approx \pi$, the longitudinal impedance of a pair of striplines is [7]

$$
\left.Z_{0}^{\|}\right|_{\mathrm{BPM}}=2 Z_{c}\left(\frac{\phi_{0}}{2 \pi}\right)^{2}\left(2 \sin ^{2} \frac{\omega \ell}{c}-i \sin \frac{2 \omega \ell}{c}\right)
$$

where $Z_{c}=50 \Omega$ is the termination impedance. The vertical dipole impedance can be expressed in terms of the longitudinal as

$$
\left.Z_{1}^{V}\right|_{\mathrm{BPM}}=\left[\frac{Z_{0}^{\|}}{\omega}\right]_{\mathrm{BPM}} \frac{16 c}{b^{2} \phi_{0}^{2}} \sin ^{2} \frac{\phi_{0}}{2} .
$$

There are 205 sets of vertical BPMs and 205 sets of horizontal BPMs. The total contribution to the vertical dipole impedance of all BPMs is shown in Fig. 4. We see that $\left.\mathcal{I m}_{1} Z_{1}^{V}\right|_{\mathrm{BPM}} \approx$ $-i 10.25 \mathrm{M} \Omega / \mathrm{m}$ below $10 \mathrm{MHz}$, which will give a slight cancellation to the space-charge impedance. The real part exhibits maximum at $\left.\mathcal{R e} Z_{1}^{V}\right|_{\mathrm{BPM}} \approx 7.43 \mathrm{M} \Omega / \mathrm{m}$ near $f=180 \mathrm{MHz}$ (revolution harmonic $n_{\mathrm{BPM}} \sim 2000$ ). However, the driving strength of transverse instability, 
$U+i V$ in the dispersion relation of Eq. (2.19), is proportional to

$$
\text { Driving force } F \propto \frac{\operatorname{Re} Z_{1}^{V}}{\left|S_{y}\right|} \text {, }
$$

where $S_{y}$ is the effective chromaticity. At zero chromaticity, $\xi_{y}=0$, the driving force of the BPM peak impedance is therefore about

$$
F=\frac{7.43}{\left|\eta n_{\mathrm{BPM}}\right|} \mathrm{M} \Omega / \mathrm{m}=0.422 \mathrm{M} \Omega / \mathrm{m}
$$

At the chromaticity of $\xi_{y}=-2.0$, the effective chromaticity is $S_{y}=15.0$, giving the driving force $F=0.497 \mathrm{M} \Omega / \mathrm{m}$. Compared with the minimum driving force of $F=4.29 \mathrm{M} \Omega / \mathrm{m}$ derived from resistive-wall impedance in Eq. (3.5), we conclude that the BPMs do not contribute to the vertical instability observed on June 9 for revolution harmonic less than $\sim 70$. It should not drive any significant vertical instability to the beam even at $\sim 180 \mathrm{MHz}$ when the real part of the BPM impedance assumes its maximum.

At low frequencies, $\omega \ell / c \ll 1$, the BPMs do have a constant inductive contribution of

$$
\left.Z_{1}^{V}\right|_{\mathrm{BPM}}=-i \frac{16 M Z_{c} \ell}{\pi^{2} b^{2}}=-i 10.3 \mathrm{M} \Omega / \mathrm{m}
$$

for $M=205$ sets of BPMs. This will counteract the space-charge impedance slightly.

\subsection{RF Cavities}

There are four $50 \Omega$ broadband ferrite-loaded rf stations. [8] The amplifiers are of $3.5 \mathrm{~kW}$ from $10 \mathrm{kHz}$ to $100 \mathrm{MHz}$, capable of supplying a total of $\pm 2 \mathrm{kV}$. The rf waveform generated is determined by the amplitude and phase of each of the 588 revolution harmonics.

Each station consists of a $12.5^{\prime \prime}$ diameter water-cooled outer aluminum shell, a $5^{\prime \prime}$ diameter aluminum inner conductor, and a $4^{\prime \prime}$ diameter stainless steel beam pipe with a $1^{\prime \prime}$ ceramic gap which is electrically connected to the cavity with beryllium-copper finger stock. The cavity is filled with $2511.5^{\prime \prime}$ OD by $6^{\prime \prime}$ ID by $1^{\prime \prime}$ thick Mn-Zn ferrite cores (MN60) and three $10^{\prime \prime}$ OD by $6^{\prime \prime}$ ID by $1^{\prime \prime}$ thick Ni-Zn ferrite cores (CMD10). The ferrite cores are aircooled, spaced by $0.5^{\prime \prime}$, and supported by Kapton spacer blocks. A $60 \Omega$ resistor is connected directly across the cavity gap and to the inner conductor at the gap by $1^{\prime \prime}$ wide by $4^{\prime \prime}$ long copper straps. A schematic drawing as shown in the top plot of Fig. 5. 

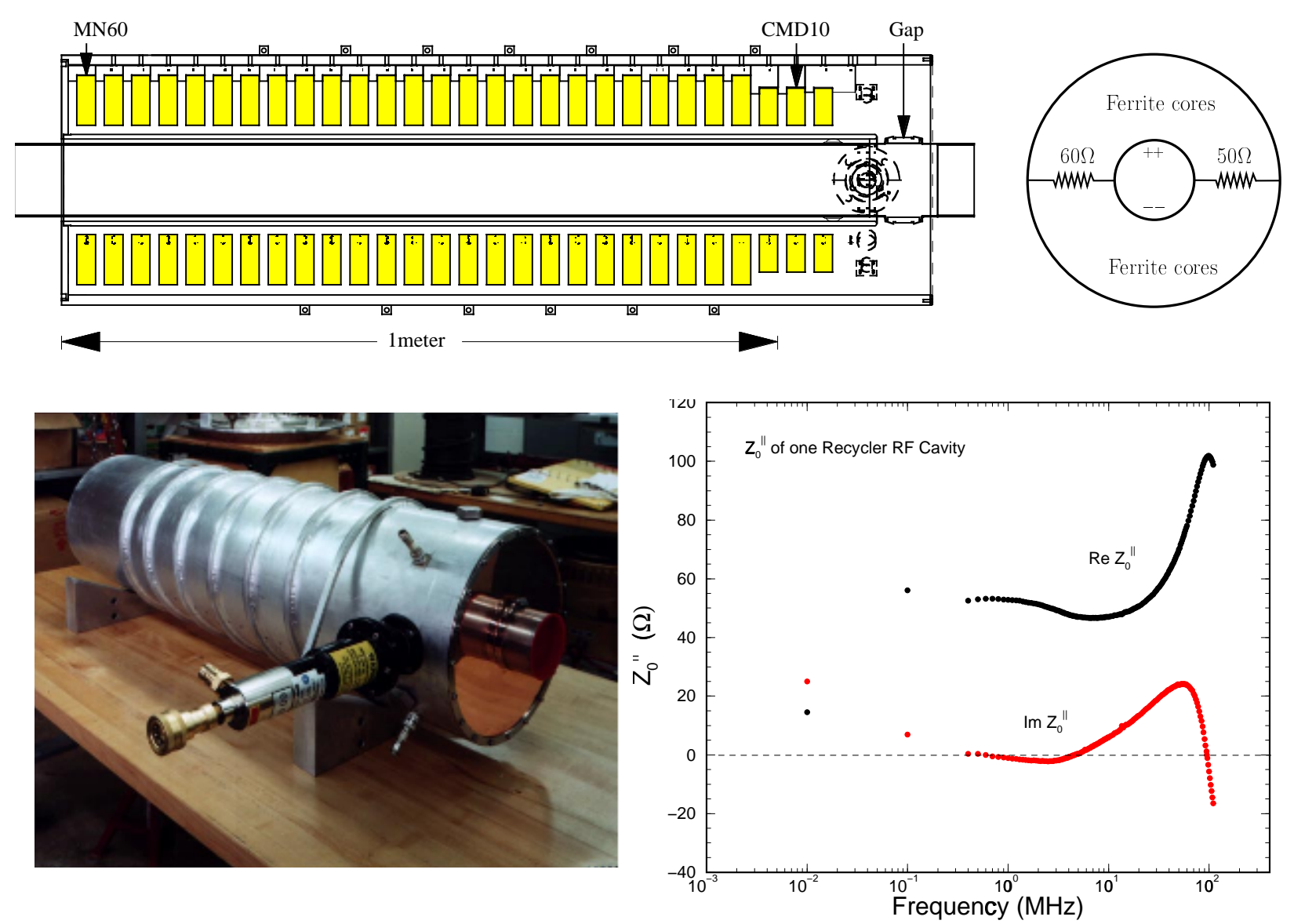

Figure 5: (color) Top: Schematic drawing of a Recycler wideband rf cavity, showing the 24 MN60 ferrite cores, the 3 CMD10 ferrite cores and its cross section. Bottom left: A photo of the cavity with $60 \Omega$ resistor protruding horizontally. Bottom right: $\operatorname{Re} Z_{0}^{\|}$and $\operatorname{Im} Z_{0}^{\|}$of the rf cavity measured by Dye and Wildman, with the $50 \Omega$ not connected to the amplifier.

The outer and inner aluminum shells form a ferrite loaded coaxial transmission line. The monopole impedance $Z_{0}^{\|}$seen by a particle beam consists of the $60 \Omega$ resistor and the copper strap in parallel with the input impedance of the coaxial transmission line. This understanding [9] can reproduce most of the characteristics of the impedance measured by Dye and Wildman. [8] The measurement, shown in the bottom right plot of Fig. 5, indicates that the monopole impedance is mostly real and that $\operatorname{Re} Z_{0}^{\|}$is mostly constant at $50 \Omega$ below $\sim 50 \mathrm{MHz}$ and starts rolling off to zero below $100 \mathrm{kHz}$, roughly the revolution frequency of the Recycler Ring. For this reason, we see a $\operatorname{Re} Z_{0}^{\|} / n \sim 50 \Omega$ near revolution frequency. In the measurement the $50 \Omega$ input resistor was not connected to the amplifier. If connected we expect $\operatorname{Re} Z_{0}^{\|} / n$ becomes $25 \Omega$. Thus, for four cavities, we expect $\operatorname{Re} Z_{0}^{\|} / n \approx 100 \Omega$ near revolution frequency. If we use the Panofsky-Wenzel-like formula to estimate the transverse 
impedance, we obtain

$$
\operatorname{Re} Z_{1}^{\perp}=\frac{2 c}{b^{2}} \frac{\operatorname{Re} Z_{0}^{\|}}{\omega} \approx 37 \mathrm{M} \Omega / \mathrm{m}
$$

where $b=2^{\prime \prime}$ has been used as the radius of the beam pipe at the cavities. This transverse impedance appears to be larger than the contribution from the resistive wall.

The situation becomes worse if we take into account of the $50 \Omega$ input resistor and the $60 \Omega$ output resistor. These resistors are in the horizontal position, while the image dipole current clusters mostly in the top and bottom of the beam pipe, as illustrated in the crosssectional view in the top right plot of Fig. 5. At low frequencies, it is not difficult to imagine that the dipole currents will not be able to see these two resistors at all. As a result, the dipole current just flows into the upper and lower parts of the cavity and experiences two heavily ferrite-loaded transmission line. The implication is that $\operatorname{Re} Z_{1}^{V}$ will be very much larger than what was estimated in Eq. (3.15) above.

However, Sacherer [10] (and also many other authors) pointed out that the PanofskyWenzel-like relation between the monopole impedance and the dipole impedance is strictly valid only for the resistive wall impedance of a round beam pipe provided that the frequency is not too low. A simple case of a deviation is shown in Fig. 6, namely a high impedance interruption in the vacuum chamber, for example a ceramic tube with a resistive coating. The monopole impedance $Z_{0}^{\|}$is increased, but not the dipole impedance $Z_{1}^{\perp}$, because the differential image current can avoid the high impedance region. The dipole impedance is increased only at very high frequencies where the wavelength becomes comparable to the interruption length or the pipe circumference, or in other words, where the added inductance due to the more circuitous path begins to contribute. Roughly speaking, this occurs around the cutoff frequency of the beam pipe, $\sim 400 \mathrm{MHz}$ for the present $2^{\prime \prime}$-radius beam pipe of the cavities. Thus below $\sim 400 \mathrm{MHz}$, we expect the dipole current to flow, after the passage of the beam particles, vertically near the gaps of the cavities and annihilates at the $50-\Omega$ input and 60- $\Omega$ output resistors, instead of flowing into the cavities. In other words, below $\sim 400 \mathrm{MHz}$, we do not expect the cavities to contribute significantly to the dipole impedance.

In conclusion, the vertical dipole impedance of the Recycler Ring is dominated by the resistive wall and space charge below $\sim 400 \mathrm{MHz}$. In addition, we may allow $\sim-i 30 \mathrm{M} \Omega / \mathrm{m}$ of inductive impedance at low frequencies to account for the BPMs, size variations, and other discontinuities of the vacuum chamber. 


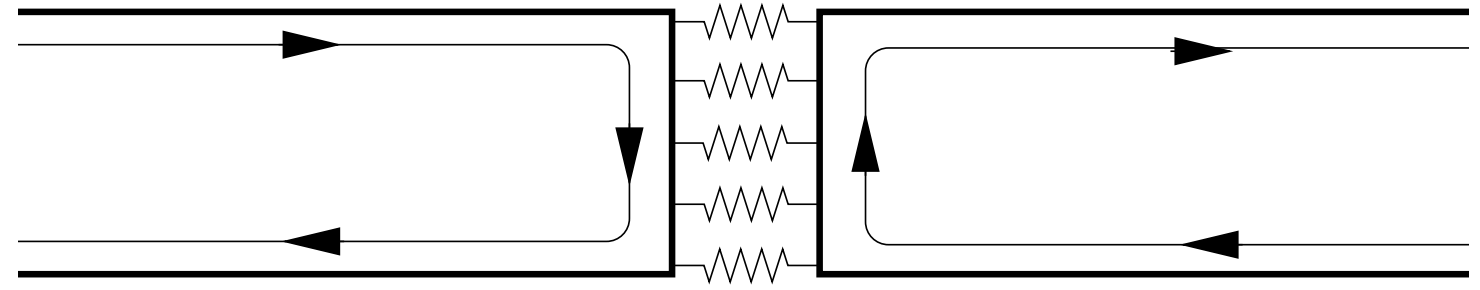

Figure 6: Illustration showing an interruption in the vacuum chamber increases the monopole impedance $Z_{0}^{\|}$but not the dipole impedance $Z_{1}^{\perp}$.

\section{Application to Instability of Proton Beam}

A proton beam consisting of 13 bunches at 4-turn injection was injected into the Recycler Ring from the Main Injector on July 8, 2004. [4] Initially, the beam intensity was $79 \times 10^{10}$. It was debunched and scraped to $43.9 \times 10^{10}$ using the scraper Top Jaw so that the $95 \%$ horizontal and vertical emittances were about $\epsilon_{N 95}=6 \pi \mathrm{mm}-\mathrm{mr}$. The skew quadrupole SQ408 was set to zero current to simulate the period from December 2003 to June 122004. The quadrupole-correction loop (QCL) was turned off to enhance the effect of the Main Injector ramping. Finally when the vertical chromaticity was reduced from $\xi_{y}=-2$ to zero, an instability of the beam was observed, with an about 6-fold jump of vertical emittance and a $14 \%$ beam loss, as illustrated in Fig. 7. The difference signal in Fig. 8 shows that the growth time was about 500 to 1000 turns or 11 to $56 \mathrm{~ms}$. The rms energy spread was $1 \mathrm{MeV}$ and did not change throughout the instability. The FFT in Fig 9 shows the lower betatron sidebands (red) excited very much more than the upper sidebands (blue) starting from $(1-Q)$. The amplifier connected to the monitoring BPM has been patched so that its gain is constant throughout the frequency range of observation down to $\sim 10 \mathrm{kHz}$.

At zero vertical chromaticity, the stability $U-V$ plot is shown in Fig 10 according to the dispersion relation of Eq. (2.19). The equi-growth contours are standardized to be the inverse of complex error function in this and later plots. The $(U V)$ values (or $-Z_{1}^{V}$ ) of each of the $(n-Q)$ modes are shown in circles. The proton beam should not have a transverse biGaussian distribution because (1) it had never been cooled stochastically and (2) it had been scraped heavily so that only about one half of the particles remained. We think it would be more appropriate to approximate the transverse distribution as uniform. The space-charge 
self-field impedance is therefore

$$
\left.Z_{1}^{V}\right|_{\mathrm{spch}}=i \frac{Z_{0} \nu_{y}}{\gamma \beta \epsilon_{N 95}}=i 162 \mathrm{M} \Omega / \mathrm{m}
$$

This becomes $i 103 \mathrm{M} \Omega / \mathrm{m}$ after adding $\sim-i 28.6 \mathrm{M} \Omega / \mathrm{m}$ coming from the inductive wall and another $\sim-i 30 \mathrm{M} \Omega / \mathrm{m}$ inductive impedance coming from BPMs, pump ports, cross-section variations, etc. The $(1-Q)$ mode is off-scale at $(U V)=(78.0,-281)$ and is on the contour of reduced growth rate $\mathcal{I} m z_{1}=44.0$ units, which corresponds to an actual growth rate of

$$
\frac{1}{\tau}=\sqrt{2} \sigma_{\delta}\left|S_{y}\right| \omega_{0} \operatorname{Im} z_{1}=19.6 \mathrm{~s}^{-1},
$$

or a growth time of $51.2 \mathrm{~ms}$. The $(2-Q)$ excitation has a growth rate of $11.9 \mathrm{~s}^{-1}$ or a growth time of $84.1 \mathrm{~ms}$. They agree approximately with the observed growth times of $75 \mathrm{~ms}$ and $105 \mathrm{~ms}$ for these two modes as depicted in Fig. 12. At $\xi_{y}=-0.3$, we see that $(1-Q)$ is at $(U V)=(1.27,-4.59)$ near the 0.65 -unit growth contour and is at $(U V)=(0.77,-2.20)$ near the 0.3-unit growth contour when $\xi_{y}=-0.5$. When $\xi_{y}=-0.775$, all modes are inside the stability region.

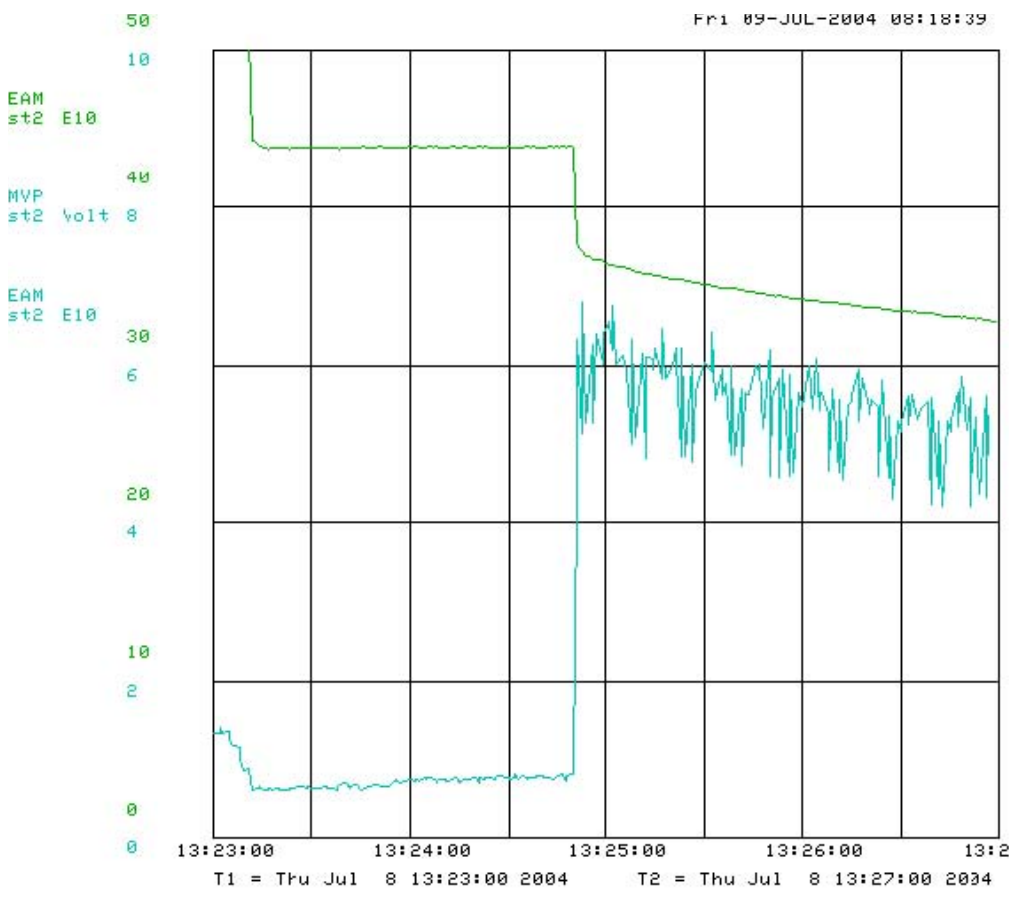

Figure 7: (color) A vertical instability was observed in a coasting proton beam of intensity $43.9 \times$ $10^{10}$ when it was heavily scraped to transverse emittances of $\sim 6 \pi \mathrm{mm}-\mathrm{mr}$ using the Top Jaw, reducing the vertical chromaticity from $\xi_{y}=-2$ to zero, turning off the quadrupole-control loop and skew quadrupole SQ408. 


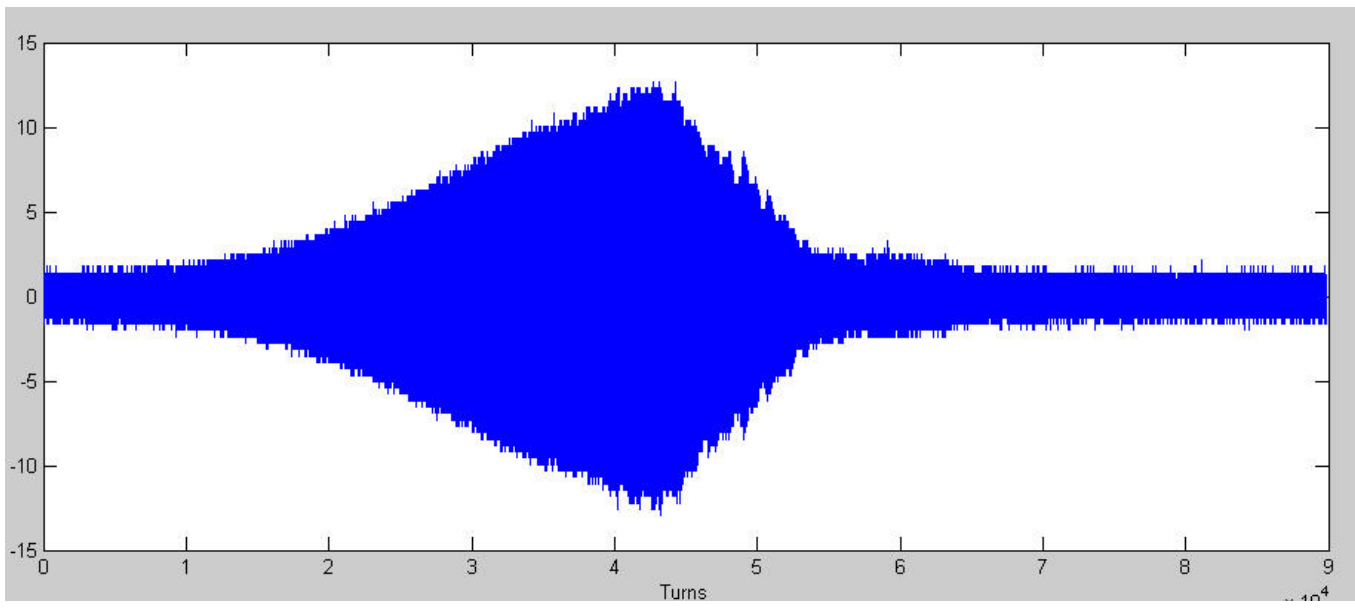

Figure 8: (color) Difference signals sampling at roughly $8 \mathrm{MHz}$ for a period of $9 \times 10^{4}$ revolution turns during the induction of transverse instability in the coasting proton beam.

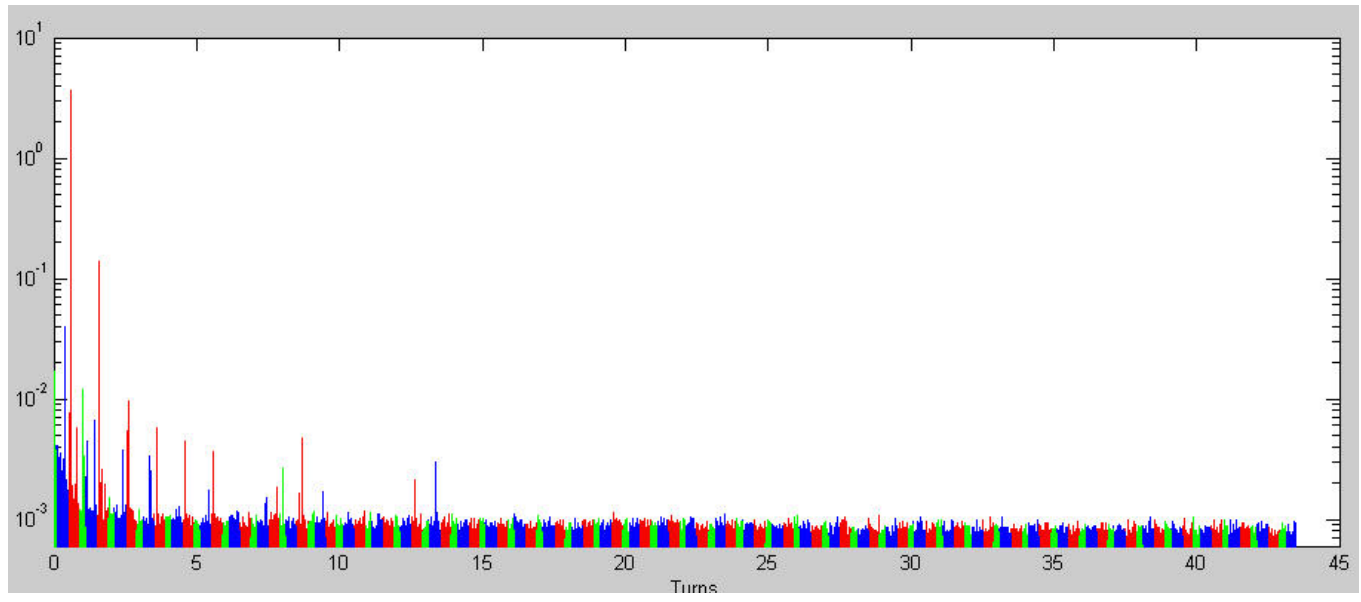

Figure 9: (color) FFT of 1048576 samples between turn 30,000 to 42,000 of the difference signals in Fig. 8. The lower sidebands are in red, the upper sidebands are in blue, and the harmonic lines are in green. The $(1-Q)$ line is clearly dominant. The amplifier gain is constant in the frequency range of observation down to $\sim 10 \mathrm{kHz}$ 


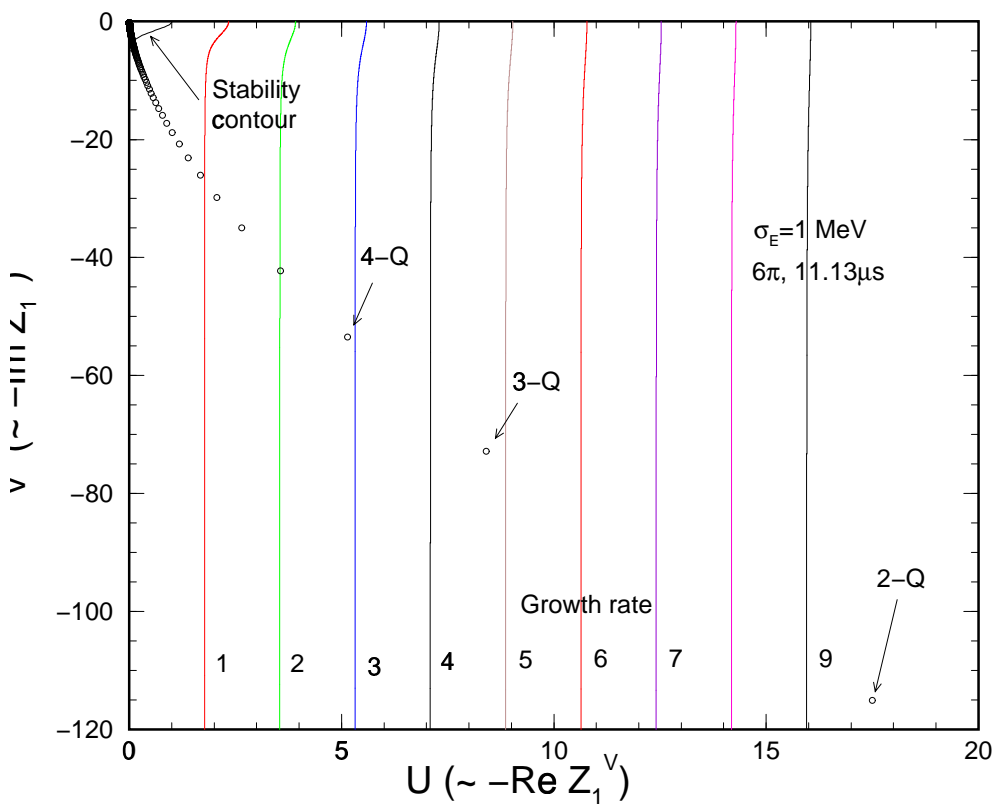

Figure 10: (color) Plot showing the equi-growth contours in the $U-V$ (normalized $-Z_{1}^{V}$ ) plane. The $(U, V)$ values of each betatron excitation $(n-Q)$ with $n=1,2,3, \cdots$ are shown as circles for $\xi_{y}=0$. The $(1-Q)$ excitation is off-scale at $(U V)=(78.0,-281)$.

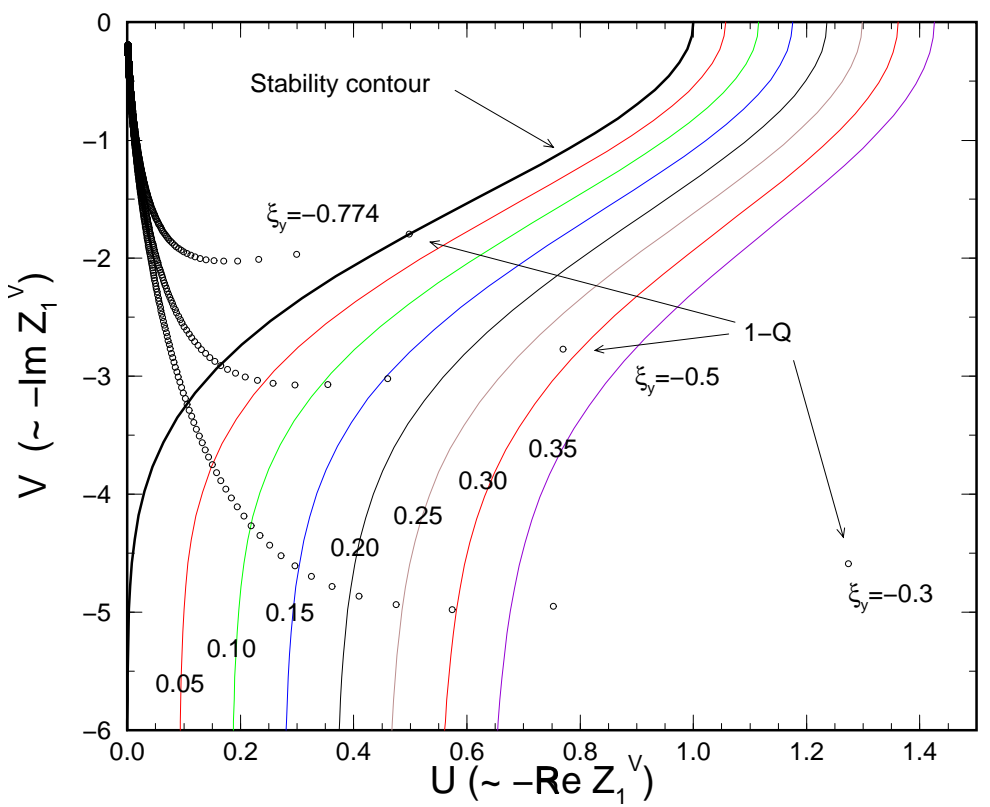

Figure 11: (color) Same as Fig. 10 at $\xi_{y}=-0.3,=-0.5$, and -0.775 . At $\xi_{y}=-0.774$, all modes are inside the stable region. 


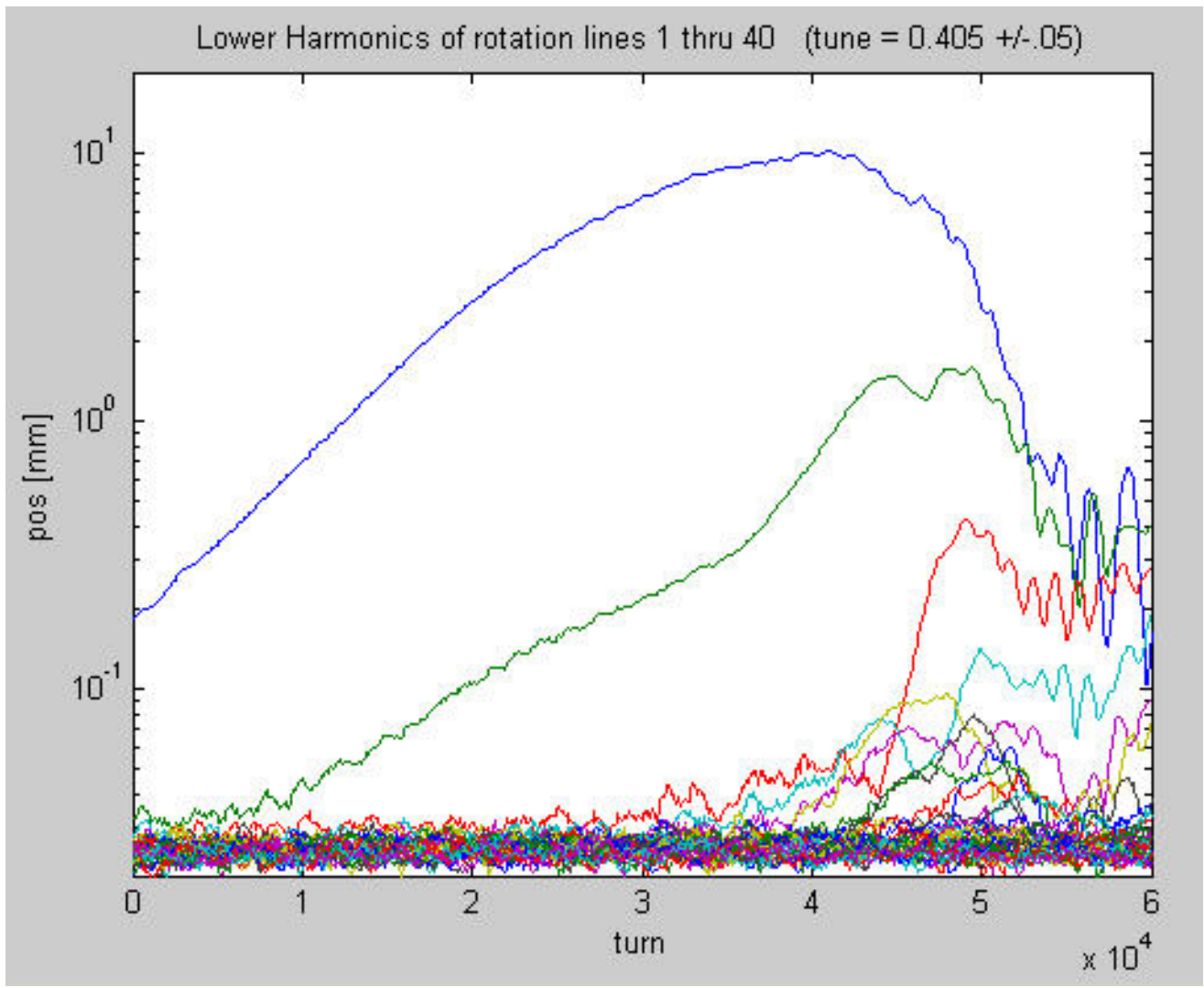

Figure 12: (color) The amplitudes of the first 40 lower betatron sidebands during the induced instability of the proton beam on July 8, 2004. The largest one is the $(1-Q)$ sideband and the next one is the $(2-Q)$ sideband.

Although the $(U V)$ values of the $(1-Q)$ mode differ so much at $\xi_{y}=0$ and -0.3 , the computed actual growth rates are, respectively, $19.6 \mathrm{~s}^{-1}$ and $17.8 \mathrm{~s}^{-1}$, and are comparable. The physical reason is suppression of Landau damping by space charge and this will be explained in detail in the next section. As illustrated in Fig. 13, the growth rates of the lowfrequency modes are nearly independent of chromaticity. Actually the effect of chromaticity is important only when the excitation is near stability. For example, the first stable mode at $\xi_{y}=0$ is $(60-Q)$. However, $\xi_{y}=-0.1$ stabilizes the mode at $(50-Q), \xi_{y}=-0.2$ stabilizes the mode at $(39-Q), \xi_{y}=-0.3$ stabilizes the mode at $(29-Q)$.

The independence of the growth rate on chromaticity suggests that it is possible to derive a formula of the growth rate when the excitation is far from stability. The complex 


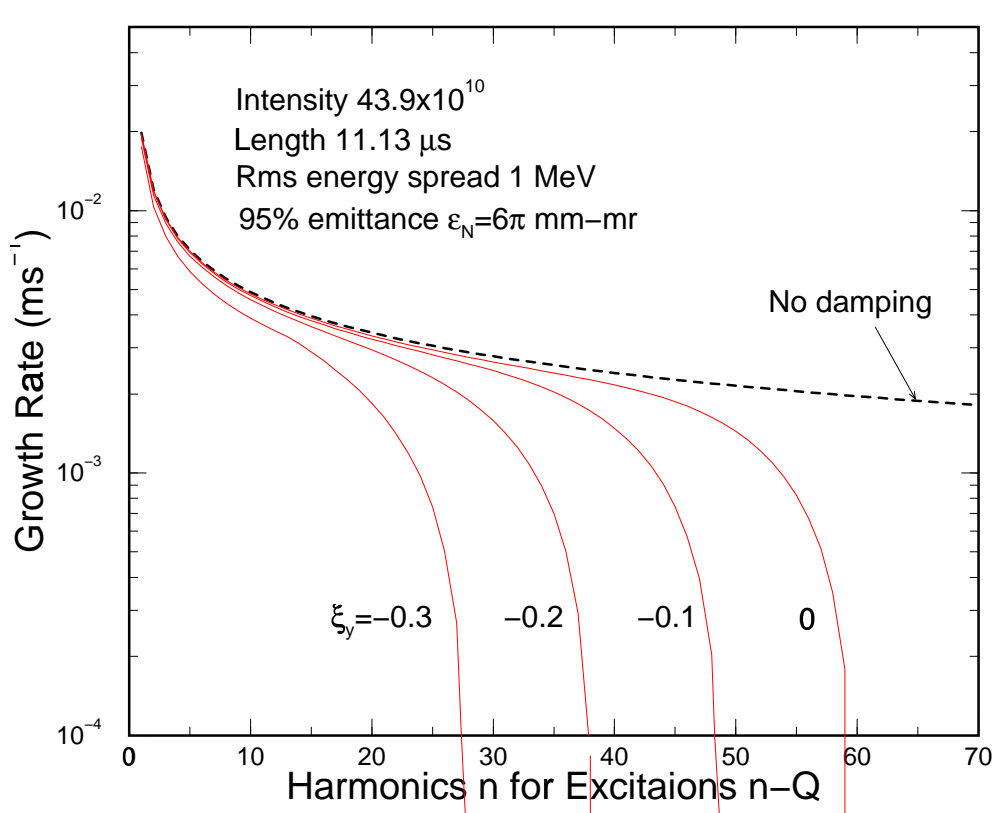

Figure 13: (color) Growth rates for excitation $(n-Q)$ at vertical chromaticity $\xi_{y}=0,-0.1,-0.2$, and -0.3 . The simple theoretical expectation is shown in dashes, which is the growth rate without Landau damping, and is in excellent agreement when the excitations are far from stability.

error function has the asymptotic behavior

$$
\frac{1}{w\left(z_{1}\right)}=-i \sqrt{\pi} z_{1}\left[1+\mathcal{O}\left(\frac{1}{\left|z_{1}\right|^{2}}\right)\right]
$$

provided that $\left|\arg \left(i z_{1}\right)\right|<\frac{3}{4} \pi$. This has been demonstrated obviously by the equi-growth contours in Fig. 13. For example, the $\operatorname{Im} z_{1}=1$ contour (growth rate of 1 unit) approaches $\operatorname{Re} w\left(z_{1}\right)^{-1}=\sqrt{\pi} \operatorname{Im} z_{1}=\sqrt{\pi}$. Taking the real part of the dispersion relation [Eq. (2.19)] and using the definitions of $(U, V)$ and $z_{1}$ [Eqs. (2.18)], we obtain

$$
-\frac{e I_{0} \beta^{2} c \mathcal{R e} Z_{1}^{V}}{4 \sqrt{2 \pi} \nu_{y} \omega_{0} \sigma_{E}\left|S_{y}\right|}=\frac{\sqrt{\pi} \operatorname{Im} \Omega}{\sqrt{2}\left|S_{y}\right| \omega_{0} \sigma_{\delta}} .
$$

We see that the chromaticity factors cancel arriving at the simple expression for the growth rate,

$$
\frac{1}{\tau}=\operatorname{Im} \Omega=-\frac{e I_{0} c \operatorname{Re} Z_{1}^{V}}{4 \pi \nu_{y} E} .
$$

This theoretical expectation is plotted in Fig. 13 as dashes. We see that it agrees very well with the exact numerical solution and shows significant deviations only when the excitations are close to stability. Notice the negative sign on the right-side of Eq. (4.5). It reminds us that instabilities are driven only by the slow waves at negative frequencies where $\operatorname{Re} Z_{1}^{V}<0$. 
Actually, the expression in Eq. (4.5) is exactly the growth rate without Landau damping, easily derivable from the dispersion relation, for example, Eq. (2.10).

\subsection{Space-Charge Effects}

The large growth rate of $19.6 \mathrm{~s}^{-1}$ for the $(1-Q)$ excitation is a result of the large spacecharge impedance, although it is the real part of the impedance that drives the growth. We mentioned in Fig. 11 that the $(1-Q)$ excitation is at $(U, V)=(1.27,-4.59)$ when the chromaticity is $\xi_{y}=-0.3$. If there were no space-charge impedance, this excitation would be at $(U, V)=(1.27,0)$, where we have neglected, for convenience, the reactive part of the resistive-wall impedance. To move it inside the stability curve will only require $\xi_{y} \approx-0.3 \times 1.27=-0.38$, since the $V$ value is proportional to $\operatorname{Re} Z_{1}^{V} /\left|S_{y}\right|$. The tune spread required to Landau-damp the instability is therefore $\sigma_{\Delta \nu_{y}}=\left|\xi_{y} \sigma_{\delta}\right|=4.3 \times 10^{-5}$. This rms tune spread is reasonable, because it corresponds to an rms angular betatron frequency spread of $\sigma_{\omega_{\beta}}=24 \mathrm{~Hz}$ while the growth rate of the $(1-Q)$ excitation is $19.6 \mathrm{~s}^{-1}$ as indicated in Fig. 13.

Space-charge shifts the incoherent tune spread downward away from the coherent excitation and Landau damping cannot be activated. For this reason, a sizeable chromaticity is required to generate a tune spread so that the coherent excitation is again inside the incoherent spread and Landau damping becomes possible.

The incoherent self-field space-charge tune shift is related to the transverse self-field space-charge impedance by

$$
\left.\Delta \nu_{y}\right|_{\text {incoh }}=i \frac{e I_{0} R}{4 \pi \beta E \nu_{y}} Z_{1}^{V}
$$

With the self-field space-charge impedance given by Eq. (3.6) with $-i 28.7 \mathrm{M} \Omega / \mathrm{m}$ of inductive wall impedance and about $-i 30 \mathrm{M} \Omega / \mathrm{m}$ inductive part from other discontinuities in the vacuum chamber subtracted, the incoherent self-field space-charge tune shift is

$$
\left.\Delta \nu_{y}\right|_{\mathrm{spch}}=-1.26 \times 10^{-4}
$$

Because a uniform transverse distribution has been assumed, this is the self-field space-charge tune shift for every particle in the beam. According to the $U-V$ plot, a vertical chromaticity

\footnotetext{
${ }^{\ddagger}$ For the excitation of $1-Q, S_{y} \approx \xi_{y}$ because the slip factor is $\eta=-0.008815$.
} 


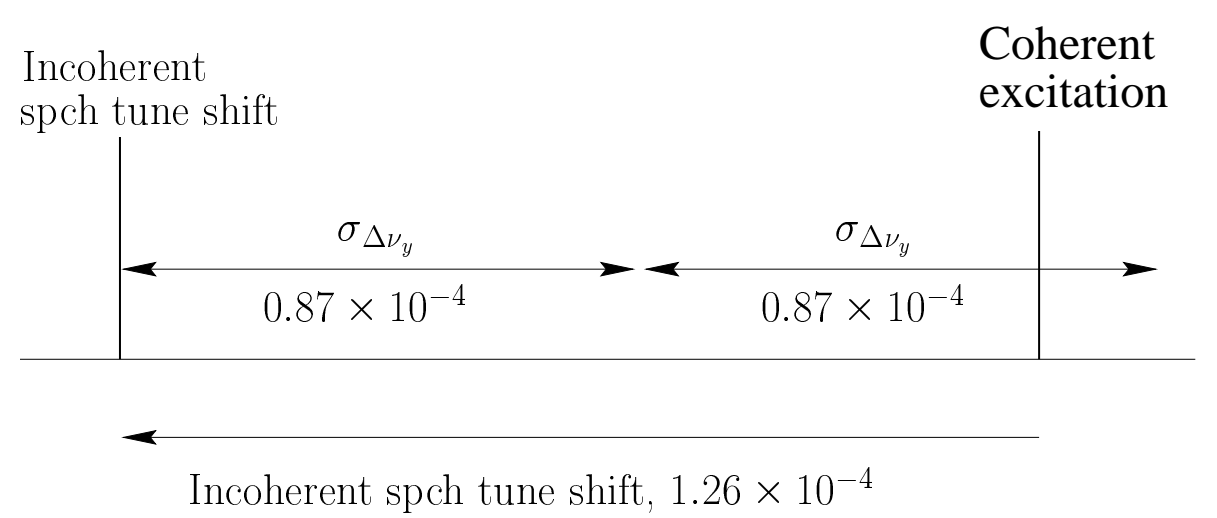

Figure 14: Incoherent tune shift is $1.26 \times 10^{-4}$ away from the coherent excitation. A chromaticity of $\xi_{y}=-0.774$ provides a rms tune spread of $\sigma_{\Delta \nu_{y}}=0.87 \times 10^{-4}$. A half spread of $2 \sigma_{\Delta \nu_{y}}$ will cover the coherent excitation and activate Landau damping.

$\xi_{y}=-0.774$ will suffice to damp transverse instability. This leads to an effective chromaticity of $S_{y}=-0.779$ for the $(1-Q)$ mode, or a rms tune spread of

$$
\sigma_{\Delta \nu_{y}}=\left|S_{y}\right| \sigma_{\delta}=0.87 \times 10^{-4} .
$$

A half spread of $\sim 2 \sigma_{\Delta \nu_{y}}=1.74 \times 10^{-4}$ is slightly larger than the incoherent self-field tune shift, and will therefore be able to cover the coherent tune line providing Landau damping. This is illustrated schematically in Fig 14 .

\section{Antiproton Instability on June 9}

Transverse instability was induced on June 9 to an antiproton beam of intensity $N_{b}=$ $30 \times 10^{10}$, length $T_{b}=3.5 \mu \mathrm{s}$, and rms energy spread $\sigma_{E}=3 \mathrm{MeV}$. When the vertical chromaticity was reduced to zero, the beam became unstable with the $95 \%$ vertical emittance jumping from $\epsilon_{N 95}=3 \pi \mathrm{mm}$-mr to $6 \pi \mathrm{mm}-\mathrm{mr}$ together with a small beam loss.

As in the case for the proton beam, we make the stability plot in the $U-V$ plane. The results are shown in Fig. 15 for vertical chromaticity $\xi_{y}=-2,-1$, and -0.5 . At $\xi_{y}=-2$, the plot shows that stability is maintained only for the betatron modes $(n-1)$ with $n \geq 13$, which is in contradiction to the daily operation, because antiproton beam of this intensity, length, energy spread, and emittances has always been stable at such chromaticity. It is possible that there are other means of Landau damping that have not been considered here. 


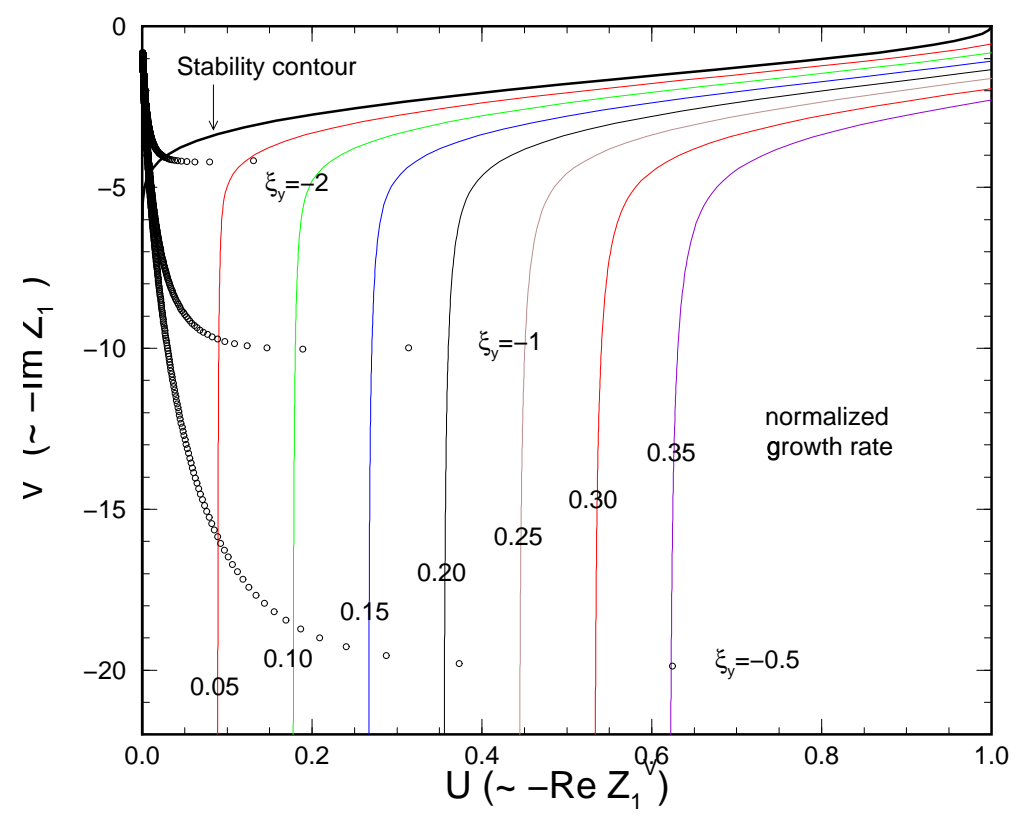

Figure 15: (color) Plot showing the equi-growth contours in the $U-V$ (normalized $-Z_{1}^{V}$ ) plane. The $(U, V)$ values of each betatron excitation $(n-Q)$ with $n=1,2,3, \cdots$ are shown as circles for $\xi_{y}=-2,-1$, and -0.5 . Here, with $\xi_{y}=-2$, stability is maintained only for modes equal to or higher than $(13-Q)$. To stabilize all the modes, a chromaticity $\xi_{y}=-2.26$ is required.

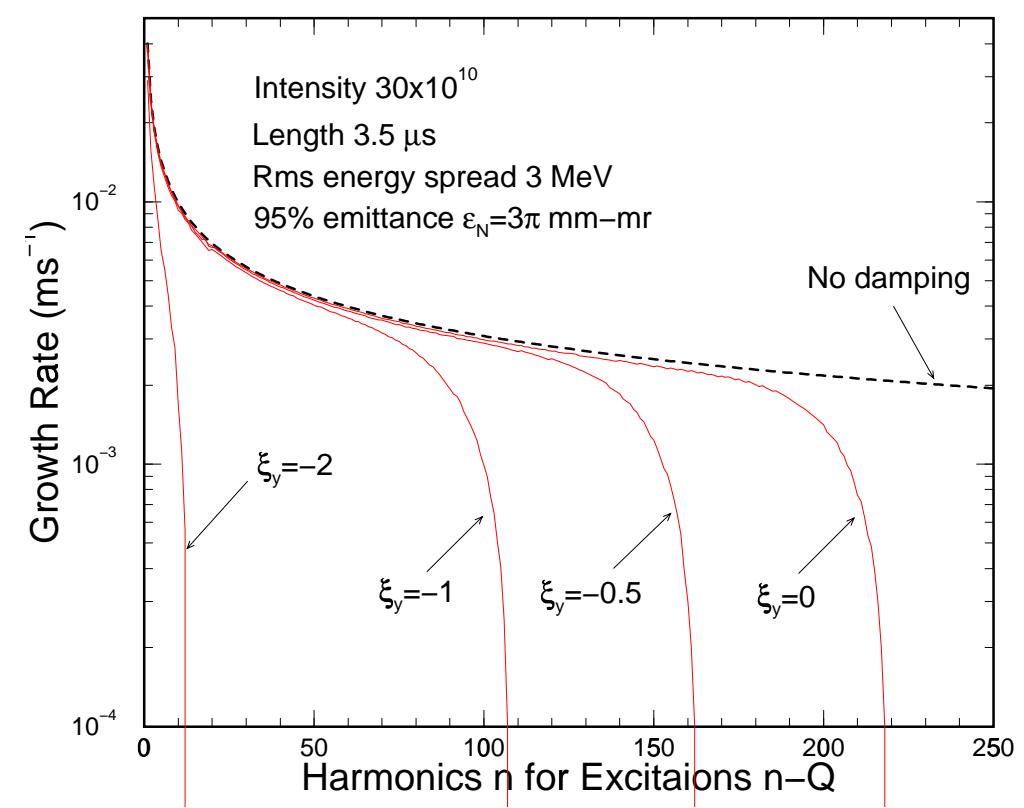

Figure 16: (color) Growth rates for excitation $(n-Q)$ at vertical chromaticities $\xi_{y}=0,-0.5,-1$, and -2 . The simple theoretical expectation, which is the growth rate without Landau damping, is shown in dashes, which is the growth rate without Landau damping, and is in excellent agreement when the excitations are far from stability. 
According to the present dispersion relation, we require a vertical chromaticity of $\xi_{y}=-2.26$ to stabilize all the betatron modes.

The growth rates of the lower betatron sidebands are depicted in Fig. 16. At zero chromaticity, all $(n-Q)$ with $n<220$ are unstable. However, we see in Fig. 2 that only those modes for $n \lesssim 70$ are unstable, another indication showing that the antiproton beam was actually more stable than the theoretical prediction. By the way, the growth rate without Landau damping for this antiproton beam is $\tau^{-1}=40.2 \mathrm{~s}^{-1}$ agreeing with observation.

We also see in Fig. 2 that the $(1-Q)$ mode has not been excited as much as the $(2-Q)$ mode, although the resistive-wall impedance at $(1-Q)$ is $\sqrt{\left(2-\left[\nu_{y}\right]\right) /\left(1-\left[\nu_{y}\right]\right)}=1.65$ times larger than the impedance at $(2-Q)$. This apparent paradox can be explained by the finite length of the beam. The beam of length $T_{b}=3.5 \mu$ s can support a wave of maximum half wavelength $3.5 \mu \mathrm{s}$. Thus the minimum frequency of excitation is $1 /\left(2 T_{b}\right)=143 \mathrm{kHz}$. The $(1-Q)$ mode is at $\left(1-\left[\nu_{\beta}\right]\right) f_{0}=52.5 \mathrm{kHz}$ and is therefore harder to excite. On the other hand, the $(2-Q)$ mode is at $142 \mathrm{kHz}$ and is therefore easier to excite.

\section{$5.1 \quad$ Space-Charge Effects}

The large growth rate of $40.2 \mathrm{~s}^{-1}$ for the $(1-Q)$ excitation at zero chromaticity is a result of the large space-charge impedance, although it is the real part of the impedance that drives the growth. The impedance of the $(1-Q)$ excitation has the value $(U, V)=$ $(52.77,-1680)$ at zero chromaticity. If there were no space-charge impedance, this excitation would be at $(U, V)=(52.77,0)$, where we have neglected, for convenience, the reactive part of the resistive-wall impedance. To move it inside the stability curve will only require $S_{y} \approx 52.77 \eta\left[\nu_{\beta}\right]=-0.27$. The tune spread required to Landau-damp the instability is therefore $\sigma_{\Delta \nu_{y}}=\left|\xi_{y} \sigma_{\delta}\right|=0.923 \times 10^{-4}$. This rms tune spread is reasonable, because it corresponds to an rms angular betatron frequency spread of $\sigma_{\omega_{\beta}}=52 \mathrm{~Hz}$ while the growth rate of the $1-Q$ excitation is $40 \mathrm{~s}^{-1}$.

Space-charge shifts the incoherent tune spread downward away from the coherent excitation and Landau damping cannot be activated. For this reason, a nominal chromaticity of $\xi_{y} \approx-2.26$ is required to generate the tune spread $\sigma_{\Delta \nu_{y}}=\left|\xi_{y} \sigma_{\delta}\right|=7.59 \times 10^{-4}$ so that the coherent excitation is again inside the incoherent spread and Landau damping becomes possible. 
The incoherent self-field space-charge tune shift is

$$
\left.\Delta \nu_{y}\right|_{\mathrm{spch}}=i \frac{e I_{0} R}{4 \pi \beta E \nu_{y}} Z_{1}^{V}=22.7 \times 10^{-4},
$$

where we have used $\operatorname{Im} Z_{1}^{V}=i 912.1 \mathrm{M} \Omega / \mathrm{m}$, which is equal to the sum $\left.\operatorname{Im} Z_{1}^{V}\right|_{\mathrm{spch}}=$ $i 970.9 \mathrm{M} \Omega / \mathrm{m},\left.\operatorname{Im} Z_{1}^{V}\right|_{\text {wall }}=-i 28.7 \mathrm{M} \Omega / \mathrm{m}$ for the resistive wall at the $(1-Q)$ mode, and Im $\left.Z_{1}^{V}\right|_{\text {other }}=-i 30.0 \mathrm{M} \Omega / \mathrm{m}$ for other inductive effects of the vacuum chamber. The above is the maximum small amplitude tune shift for particles at the center of the beam. For all other particles in the beam with bi-Gaussian transverse distribution, this self-field space-charge tune shift has a distribution skewed towards the maximum as illustrated in Fig. 17. For our beam, the average incoherent tune shift is $-22.7 \times 10^{-4} \times 0.6334=-14.4 \times 10^{-4}$. At the chromaticity $\xi_{y}=-2.26$, the $2 \sigma$ 's point falls on the tune shift $-14.4 \times 10^{-4}+2 \times 7.59 \times 10^{-4}=$ $+0.83 \times 10^{-4}$. Thus the spread in tune will definitely cover the coherent excitation line and therefore the excitation can be Landau-damped.

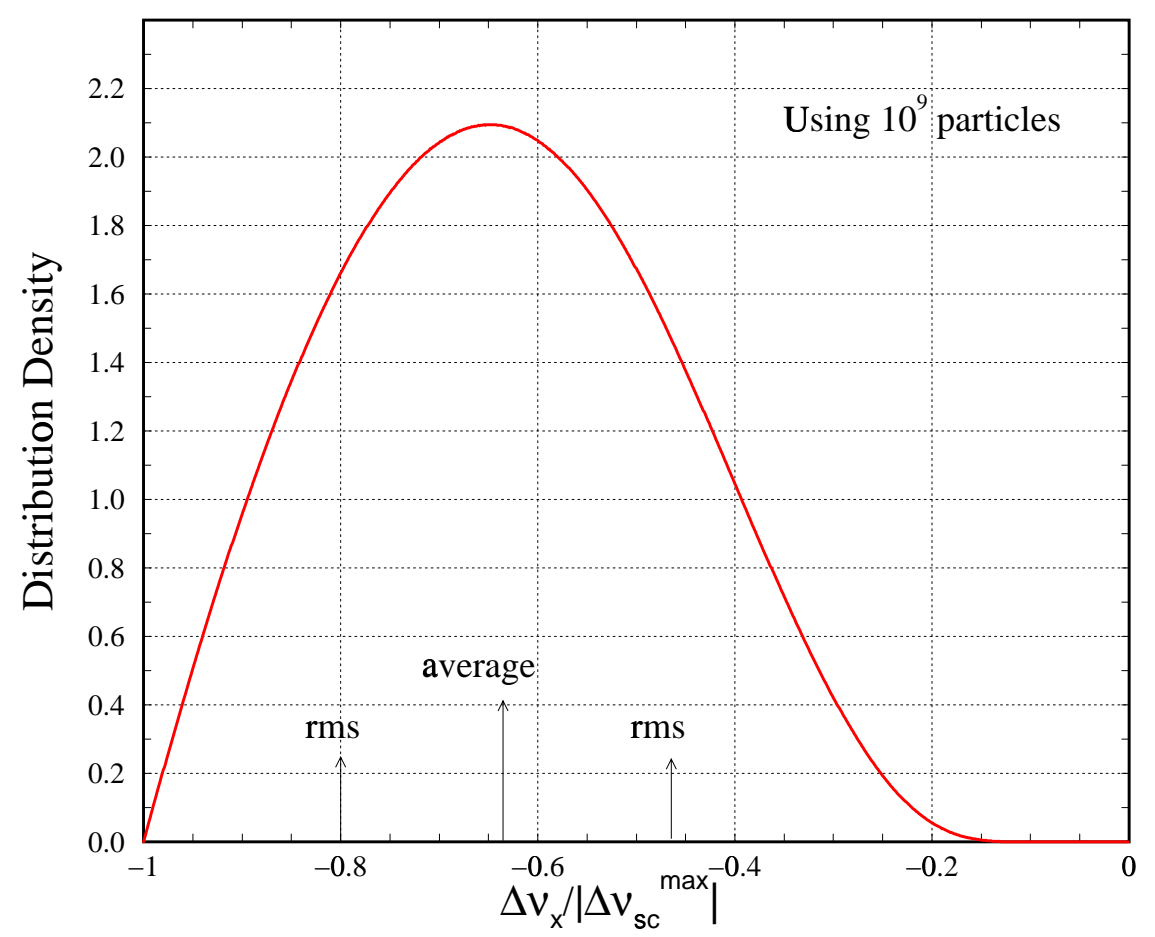

Figure 17: Distribution density of particles with incoherent horizontal space-charge tune shift $\Delta \nu_{x}$, in units of the maximum $\left|\Delta \nu_{s c}^{\max }\right|$. Also shown are the average and rms spread which are, respectively, 0.6334 and 0.1678 of $\Delta \nu_{s c}^{\max }$. 


\section{Conclusions}

(1) Although the measured sideband spectra point to instabilities driven by the resistivewall impedance, however, the computed growth rates appear larger than observed and not so many sidebands are unstable in observation. For example, in the induced instabilities of the proton beam, only the lowest $\sim 15$ betatron sidebands were excited (Fig. 2), but the dispersion relation says 60 are unstable (Fig. 13). In the induced instabilities of the antiproton beam (Fig. 8), only the lowest $\sim 70$ betatron sidebands were excited, but the dispersion relation says 250 are unstable (Fig. 16).

There may also be possible Landau damping coming from the nonlinear lattice elements of the ring. The nonlinear betatron tune shifts from these elements can be expressed as

$$
\begin{aligned}
& \Delta \nu_{x}=\frac{\partial \nu_{x}}{\partial \epsilon_{x}} \epsilon_{x}+\frac{\partial \nu_{x}}{\partial \epsilon_{y}} \epsilon_{y}, \\
& \Delta \nu_{y}=\frac{\partial \nu_{y}}{\partial \epsilon_{x}} \epsilon_{x}+\frac{\partial \nu_{y}}{\partial \epsilon_{y}} \epsilon_{y},
\end{aligned}
$$

where $\partial \nu_{x} / \partial \epsilon_{y}=\partial \nu_{y} / \partial \epsilon_{x}$. With the emittances $\epsilon_{x, y}$ unnormalized and represented in meters, the nonlinear coefficients of the Recycler lattice including quadrupole fields, sextupole fields in the body and ends, and the feed-down due to end shims are listed in Table II from a computation using MAD. [11] With the addition of skew and normal multipole errors of the magnets, the nonlinear coefficients are found to be not much different from those listed in the table, at the most about $10 \%$ smaller. Since $\epsilon_{x} \approx \epsilon_{y}$, the tune shift is dominated by the coefficient $\partial \nu_{y} / \partial \epsilon_{x}$, both the horizontal and vertical betatron tunes are found always to shift in the negative direction independent of the chromaticity setting. In other words, particles

Table II: Nonlinear coefficients of the Recycler lattice including quadrupoles, sextupole fields in the body and ends, and the feed-down due to end shims as represented in Eq. (6.1), with the emittance in meters and unnormalized.

\begin{tabular}{ccccc}
\hline$\xi_{x} / \xi_{y}$ & $-10 /-10$ & $-1.9 /-2.3$ & $0 / 0$ & $+10 /+10$ \\
\hline$\frac{\partial \nu_{x}}{\partial \epsilon_{x}}\left(\mathrm{~m}^{-1}\right)$ & -70.7 & -70.4 & -84.6 & -28.0 \\
$\frac{\partial \nu_{y}}{\partial \epsilon_{y}}\left(\mathrm{~m}^{-1}\right)$ & +63.8 & +47.9 & +29.9 & -84.8 \\
$\frac{\partial \nu_{y}}{\partial \epsilon_{x}}\left(\mathrm{~m}^{-1}\right)$ & -140 & -360 & -426 & -719 \\
\hline
\end{tabular}


at the edge of the beam have their betatron tunes shifted downwards much more than particles at the center. This is exactly opposite to the situation of self-field space-charge tune shifts. The implication is that the total self-field space-charge tune spreads in a nonuniform transverse distribution will become smaller both horizontally and vertically, resulting in the reduction of Landau damping. Luckily, at $\xi_{x}=\xi_{y}=0$ and $\epsilon_{x N 95} \approx \epsilon_{y N 95}=3 \pi \mathrm{mm}$ $\mathrm{mr}$, the vertical betatron tune spread from nonlinear lattice fields turns out to be only $\Delta \nu_{y} \approx 1.26 \times 10^{-4}$, which amounts to about $6 \%$ of the vertical self-field space-charge tune shift [Eq. (5.1] in the antiproton-beam experiment, but is of the same order as the vertical self-field space-charge tune shift [Eq. (4.7)] in the proton-beam experiment. The conclusion is that the betatron tune spreads due to nonlinear lattice elements may help Landau damping in the proton-beam experiment, but will have very little effect on the Landau damping in the antiproton-beam experiment.

(2) We want to ask whether the space-charge impedances of the beams have been overestimated. Smaller space-charge impedances would lead to better agreement between theory and observation. For the proton beam, uniform transverse distribution has been assumed and that should give the smallest transverse space-charge impedance. For the antiproton beam, stochastic cooling makes the beam very bi-Gaussian and this has been verified by measuring the transverse beam shape using scrapers. Thus there is not much problem in the estimation of the space-charge impedances.

Some physicists argue that the self-field portion

$$
\left.Z_{1}^{V}\right|_{\mathrm{spch}}=i \frac{Z_{0} R}{\gamma^{2} \beta^{2} a^{2}}
$$

should not be included in the transverse space-charge impedance. The impedance is derived by offsetting the beam transversely by a small amount as illustrated in Fig. 18 and computing the electric and magnetic forces on the beam center from the dipole charge and current distributions set up at the edges of the beam. They argue that when oscillation amplitude is larger than the beam radius, the displaced charges will not be seen by the beam. In any case, however, the space-charge impedance should play an important role at the beginning of the instability when the transverse offset is small. Experimentally, space-charge effects are certainly significant. The observed proton beam instabilities do depend on heavy scraping, and to induce instabilities of antiproton beams, reduction in the transverse beam size by stochastic cooling is certainly required. However, we notice that

$$
\left.Z_{1}^{\perp}\right|_{\mathrm{spch}} \propto \rho(0)
$$




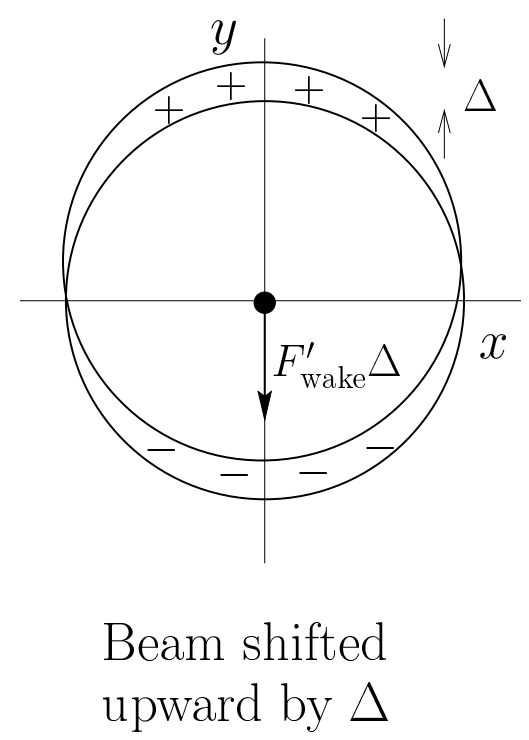

Figure 18: The transverse space-charge impedance is derived by offsetting the beam transversely and computing the dipole electric and magnetic forces on the beam center set up at the edges of the beam. If the offset $\Delta$ is larger than the beam radius, the center of the beam will no longer see the dipole electric and magnetic forces.

where $\rho(0)$ is the transverse distribution at the center of beam. Thus the transverse spacecharge impedance corresponds to the largest incoherent space-charge tune shift. Maybe something that is averaged over the beam particles would be more correct. For this, the derivation of the transverse space-charge self-field impedance should be revisited.

(3) There have been some problems concerning skew quadrupole SQ408. In May of 2004, it was discovered that SQ408 had been wired incorrectly and was not supplying the required skew-quadrupole field to counteract horizontal and vertical coupling. It was repaired near the end of June, and after that no more natural transverse instability has ever been recorded. In the induced proton instability on July 8, the turning off of SQ408 was a very essential step to excite the instability. With the SQ408 on, no instability could be induced. It is possible that the beam got jittered horizontally by the stray fields of the Main Injector and the was then coupled to the vertical to start the instability. It is also possible that with the SQ408 off, strong horizontal and vertical coupling moves the tune footprint onto some parametric resonances, which trigger the instability. In short, up to now there has not been any sound theory about the role SQ408 plays on the instabilities. 


\section{References}

[1] K.Y. Ng, Possible Ion Trapping in Recycler Ring, Proceedings of the 31st ICFA Advanced Beam Dynamics Workshop on Electron-Cloud Effect (Ecloud'04), April 19-23, 2004, Napa, CA, ed. M. Furman.

[2] All the experimental data reported in this article were gathered by members of the Fermilab Recycler Ring Department.

[3] Jim Crisp and Martin Hu, Recycler Ring Instabilities Measured on 6/9/04, 2004, (unpublished).

[4] Martin Hu and Jim Crisp, Recycler Instability Observed with Protons, 2004, (unpublished).

[5] R. Gluckstern, J. van Zeijts, B. Zotter, Coupling impedance of beam pipes of general cross section, Phys. Rev. E47 656 (1993); also reports CERN SL/AP 92-25 and 92-18;

A. Piwinski, Impedances in Lossy Elliptical Vacuum Chambers, (red) DESY Report 94068 (already known in 1993); K. Yokoya, Resistive Wall Impedance of Beam Pipes of General Cross Section, Particle Accelerators 41 (1993) 221-228.

[6] K.Y. Ng, Space-Charge Impedances of Beams with Non-Uniform Transverse Distributions, Fermilab-FN-0756, 2004.

[7] King-Yuen Ng, Impedances of stripline Beam-Position Monitors, Particle Accelerators 23, 93 (1988).

[8] J.E. Dey and D.W. Wildman, Wideband Rf System for the Fermilab Recycler Ring, PAC'99, New York, 1999, p.869.

[9] K.Y. Ng, Coherent parasitic Energy Loss of the Recycler Beam, Fermilab Internal Report FNAL-TM-2249, 2004.

[10] B. Zotter and F. Sacherer, Transverse Instabilities of Relativistic Particle Beams in Accelerators and Storage Rings, Proceedings of the First Course of the International School of Particle Accelerators of the 'Ettore Majorana' Centre for Scientific Culture, Erice 10-22 November 1976, CERN Report CERN 77-13, 1977.

[11] D. Johnson, private communication. 